\title{
Review Article \\ Closed-Form Formula of the Transverse Dynamic Stiffness of a Shallowly Inclined Taut Cable
}

\author{
Dan-hui Dan, ${ }^{1} \mathrm{Zu}-h e$ Chen, ${ }^{1}$ and Xing-fei Yan $^{2}$ \\ ${ }^{1}$ Department of Bridge Engineering, Room 711, Bridge Building, Tongji University, 1239 Siping Road, Shanghai 200092, China \\ ${ }^{2}$ Shanghai Urban Construction Design Research Institute, Shanghai 200125, China \\ Correspondence should be addressed to Dan-hui Dan; dandanhui@tongji.edu.cn
}

Received 1 December 2013; Revised 30 April 2014; Accepted 6 May 2014; Published 28 May 2014

Academic Editor: Mickaël Lallart

Copyright (c) 2014 Dan-hui Dan et al. This is an open access article distributed under the Creative Commons Attribution License, which permits unrestricted use, distribution, and reproduction in any medium, provided the original work is properly cited.

\begin{abstract}
The segmented vibration-governed equations and their general solutions for cables acted upon by intermediate transverse forces are derived by applying Hamilton's principle. Including the effects of sagging, flexible stiffness, clamped boundary conditions, and inclination angle of the cable, the element-wise dynamic stiffness for each cable segment, split into segments having unique transverse forces, is derived. By using methods from the global stiffness assembly process of FEM, the global level of the cables' dynamic equilibrium equation is obtained, and, as a result, the final closed-form formula of transverse dynamic stiffness is derived. Additionally, the corresponding analytic form, without considering sagging effects, is also obtained. Case studies are conducted on the aspects of accuracy, rationality of the distribution on the spatial field, and frequency domains of dynamic stiffness calculations. By comparison with the Guyan-based static FEM reduction method, it is shown that the result obtained from the proposed closedform solution, which includes sagging effects, is exact and rational, thus creating a powerful tool in transverse vibration analysis.
\end{abstract}

\section{Introduction}

A current trend in structure development is exceeding existing spanning limits, for which cable structures are playing an increasingly important role. With the extensive use of cable structures, the use of lateral bracing is becoming common, including the installation of intermediate turn supports, lateral dampers, and the use of cross ties, chain bar between cables, and hanger rods $[1,2]$. These elements provide transverse static forces for the main body of the cable structures and are often specially designed for improving the dynamic characteristics of the cable structures $[3,4]$. The analysis and calculation of characteristics and behaviors of cables acted upon by transverse forces exerted by these elements are also important factors in the design of a cable system. A direct relationship between the excitation and vibration response can be determined by an analysis of the dynamic stiffness, and, therefore, this particular analysis method has become an important tool in cable vibration analysis $[5,6]$.

Due to the nonlinear behavior of cables, the study on their dynamic stiffness is limited. Currently, most studies on the dynamic stiffness of cables are focused on the development of a computation method using a $4 \times 4$ matrix that describes the two-dimensional dynamic stiffness of one end of a cable, while the other end is kept fixed. Kolousek first provided the series solution to the dynamic stiffness at the free end of such cables, neglecting internal damping [7]. Davenport [8] (1959) found a closed-form dynamic stiffness matrix solution, taking damping into account and later, in 1965, developed the series solution of dynamic stiffness, which also considered damping effects [9]. In 1981, on the basis of the closed-form solution given by Davenport, Irvine proposed a simplified expression for dynamic stiffness [1]. All of these studies assumed small vibration amplitudes and a small inclination angle for the cables. In 1983, Veletsos and Darbrel studied a more accurate closed-form solution to the dynamic stiffness in the upper part of a cable under harmonic excitation, at all angles, and under a parabolic initial configuration. When damping effects were not taken into account, this closed-form solution was consistent with the series solution proposed by Veletsos and Darbre [10, 11]. In 1991, Starossek derived a dynamic stiffness matrix for cable structures with inclined 
hinges, which took sagging into consideration, and analyzed the features of its elements [12, 13]. Sarkar and Manohar proposed a calculation scheme for the dynamic stiffness matrix of cables using finite element analysis [14]. In 2001, Kim and Chang gave an approximate analytical expression of the dynamic stiffness matrix of an inclined cable with a catenary configuration [15], which surpassed the limitations of the parabolic static configuration.

Each of these studies on dynamic stiffness considered various factors based on the taut string theory, while the terms corresponding to the bending stiffness of the cables were all neglected in the passive vibration control equations. The actual cable structures are usually inclined cables with each end clamped and with relatively high bending stiffness $[16,17]$. With increased length, the effect of sag cannot be neglected $[18,19]$. A calculation algorithm of the dynamic stiffness of the cables, with all of these factors taken into account, would be very valuable to engineering practices. Furthermore, dynamic stiffness is used to describe the dynamical behavior at the ends of cables and, therefore, cannot be used in the case of a cable acted upon by transverse forces. However, in engineering practices, a majority of cable systems are acted upon by transverse forces, and, therefore, the study of transverse dynamic stiffness of cables is crucial and will contribute substantially to studies involving cable damping systems $[20,21]$.

In this study, a cable is modeled as being divided into various segments, each acted upon by a unique transverse force, while factors including sagging, bending stiffness, clamped boundary conditions, and inclination angle are all considered. The dynamic stiffness method is used to establish an equation of motion, and its general solution corresponds to a cable system acted upon by transverse forces. The dynamic displacement of each segment is used to characterize the dimensionless vibration mode function. Eventually, an analytical expression for the transverse dynamic stiffness matrix of a cable is given in a clear, simple form. The results of the study lay a foundation for further research in system vibration.

\section{General Solution for Governing Equation of Cable}

The focus of this study is on a commonly observed situation in engineering, namely, when one end of a cable is acted upon by transverse forces. As shown in Figure 1, due to the presence of transverse forces, the cable system can be discretized into cable segment 1 (from end a to end c), cable segment 2 (from end $c$ to end b), and transverse force elements (such as dampers).

As shown in Figure 1, by assuming that transverse forces do not affect the static configuration of the cable, the two cable segments can be described by the same quadratic parabolic function $y\left(x_{0}\right)$; when the cable vibrates, segments 1 and 2 follow different configurations, namely, $u\left(x_{1}, t\right)$ and $u\left(x_{2}, t\right)$. When considering the effects of sagging, bending stiffness, clamped boundary conditions, and inclination angle and ignoring the internal damping and shear forces, following

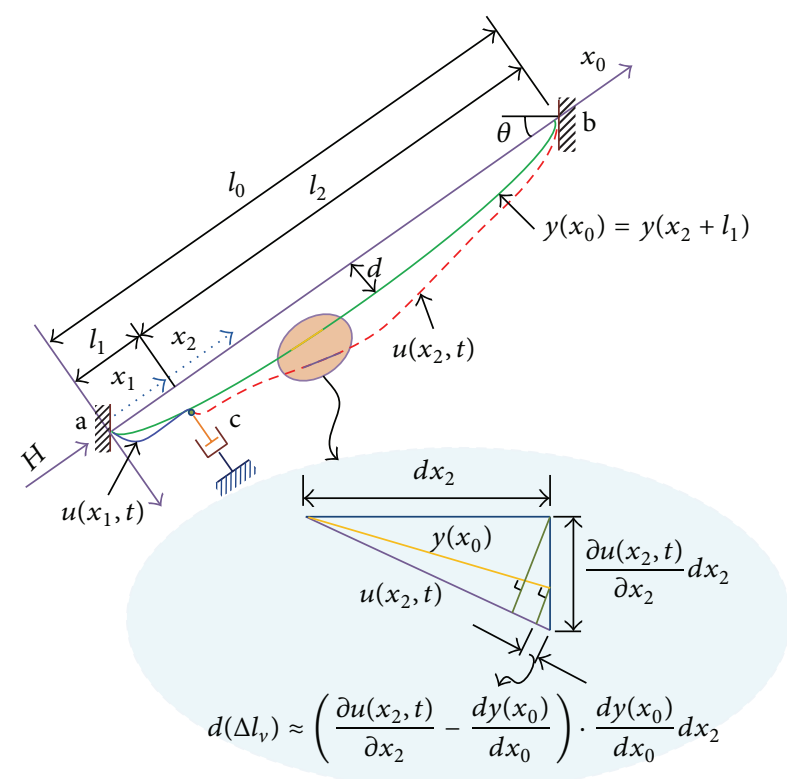

FIGURE 1: Schematic diagram of calculation of additional dynamic stress.

governing equation $[18,22]$ can be used to describe the free vibration of the two segments:

$$
E I \frac{\partial^{4} u}{\partial x_{j}^{4}}-H \frac{\partial^{2} u}{\partial x_{j}^{2}}-h_{j} \frac{d^{2} y}{d x_{0}^{2}}+m \frac{\partial^{2} u}{\partial t^{2}}=0,
$$

where $j=1,2, E I$ is the flexural rigidity, $H$ is the initial tension force, $m$ is the mass per unit length, $y$ is the configuration of the cable under static equilibrium (assuming that dampers have no impact on the static configuration), $x_{j}$ is the coordinate of each segment, and $h_{j}$ represents the additional segmented force, which is an additional cable tension generated due to the difference from the static configuration during vibration, and is equal to the product of the additional strain $\varepsilon_{j}{ }^{v}(t)$, generated during difference from the static equilibrium position, and the axial stiffness. That is,

$$
h_{j}=E A \varepsilon_{j}^{v}(t) .
$$

The additional dynamic strain $\varepsilon_{j}{ }^{v}(t)$ can be calculated using the ratio between the change in cable length $\Delta l_{j}{ }^{v}$ during vibration and the static cable length $l_{j}{ }^{e}$. Consider

$$
\varepsilon_{j}^{v}(t)=\frac{\Delta l_{j}^{v}}{l_{j}^{e}} .
$$

In (2) and (3), the superscript $v$ represents the dynamic configuration and $e$ represents static equilibrium. A similar rule is followed below.

As shown in Figure 1, in bridge projects, the angle between the tangential direction and the chord of a cable is very small for both the static and dynamic configurations. Therefore, when ignoring the higher order terms of the static and dynamic configurations, the amount of elongation $d x_{j}$ of 
a differential segment during vibration relative to that of the static configuration $d\left(\Delta l_{j}{ }^{v}\right)$ can be approximated by

$$
\begin{aligned}
d\left(\Delta l_{j}^{v}\right) & \approx\left(\frac{\partial u\left(x_{j}, t\right)}{\partial x_{j}}-\frac{d y\left(x_{0}\right)}{d x_{0}}\right) \cdot \frac{d y\left(x_{0}\right)}{d x_{0}} d x_{j} \\
& \approx \frac{\partial u\left(x_{j}, t\right)}{\partial x_{j}} \frac{d y\left(x_{0}\right)}{d x_{0}} d x_{j} .
\end{aligned}
$$

The total elongation of segment $j$ can be written as

$$
\Delta l_{j}^{v}=\int_{0}^{l_{j}} d\left(\Delta l_{j}^{v}\right)=\int_{0}^{l_{j}} \frac{\partial u\left(x_{j}, t\right)}{\partial x_{j}} \frac{d y\left(x_{0}\right)}{d x_{0}} d x_{j}
$$

With the assumption of a parabolic static equilibrium, one can use the following expression for the configuration:

$$
\begin{aligned}
y\left(x_{0}\right) & =-\frac{4 e}{l_{0}} x_{0}\left(x_{0}-l_{0}\right) \\
& =-\frac{4 e}{l_{0}}\left(x_{2}+l_{1}\right)\left(x_{2}-l_{2}\right) \\
& =-\frac{4 e}{l_{0}} x_{1}\left(x_{1}-l_{0}\right),
\end{aligned}
$$

where $e=d / l_{0}=m g l_{0} \cos \theta / 8 H$ is the sag-span ratio and $d=m g l_{0}^{2} \cos \theta / 8 H$.

Starting from (6) and noting the relationship of $x_{0}=x_{1}=$ $x_{2}+l_{1}$, we can easily get the coordinate derivative of the static configuration function $y\left(x_{0}\right)$, as follows:

$$
\begin{aligned}
\frac{d y\left(x_{0}\right)}{d x_{0}} & =-\frac{4 e}{l_{0}}\left[2 x_{0}-l_{0}\right]=-\frac{4 e}{l_{0}}\left[2 x_{1}-l_{0}\right] \\
& =-\frac{4 e}{l_{0}}\left[2 x_{2}+\left(l_{1}-l_{2}\right)\right],
\end{aligned}
$$

And then, by substituting it into (5), we have

$$
\begin{aligned}
\Delta l_{1}^{v}= & \int_{0}^{l_{1}} d\left(\Delta l_{1}^{v}\right)=\int_{0}^{l_{1}} \frac{\partial u\left(x_{1}, t\right)}{\partial x_{1}} \frac{d y\left(x_{0}\right)}{d x_{0}} d x_{1} \\
& =-\frac{4 e}{l_{0}} \int_{0}^{l_{1}} \frac{\partial u\left(x_{1}, t\right)}{\partial x_{1}}\left[2 x_{1}-l_{0}\right] d x_{1} \\
& =-\frac{4 e}{l_{0}}\left\{2 \int_{0}^{l_{1}} \frac{\partial u\left(x_{1}, t\right)}{\partial x_{1}} x_{1} d x_{1}-l_{0} \int_{0}^{l_{1}} \frac{\partial u\left(x_{1}, t\right)}{\partial x_{1}} d x_{1}\right\} \\
& =-\frac{4 e}{l_{0}}\left\{2 \int_{0}^{l_{1}} \frac{\partial}{\partial x_{1}}\left[u\left(x_{1}, t\right) x_{1}\right] d x_{1}\right. \\
& \left.-2 \int_{0}^{l_{1}} u\left(x_{1}, t\right) d x_{1}-l_{0} \int_{0}^{l_{1}} \frac{\partial u\left(x_{1}, t\right)}{\partial x_{1}} d x_{1}\right\}
\end{aligned}
$$

$$
\begin{aligned}
& =-\frac{4 e}{l_{0}}\left\{2\left[u\left(x_{1}, t\right) x_{1}\right]_{0}^{l_{1}}-2 \int_{0}^{l_{1}} u\left(x_{1}, t\right) d x_{1}\right. \\
& \left.\quad-l_{0} u\left(x_{1}, t\right)_{0}^{l_{1}}\right\} \\
& =-\frac{4 e}{l_{0}}\left\{2 l_{1} u\left(l_{1}, t\right)-2 \int_{0}^{l_{1}} u\left(x_{1}, t\right) d x_{1}-l_{0} u\left(l_{1}, t\right)\right\} \\
& =\frac{8 e}{l_{0}}\left\{\int_{0}^{l_{1}} u\left(x_{1}, t\right) d x_{1}+\left(0.5 l_{0}-l_{1}\right) u\left(l_{1}, t\right)\right\} .
\end{aligned}
$$

Similarly, we can get the expression of $\Delta l_{2}{ }^{\nu}$ :

$$
\begin{aligned}
& \Delta l_{1}^{v}=\frac{8 e}{l_{0}}\left[\int_{0}^{l_{1}} u\left(x_{1}, t\right) d x_{1}+\left(0.5 l_{0}-l_{1}\right) u\left(\left.x_{1}\right|_{=l_{1}}, t\right)\right] \\
& \Delta l_{2}^{v}=\frac{8 e}{l_{0}}\left[\int_{0}^{l_{2}} u\left(x_{2}, t\right) d x_{2}-\left(0.5 l_{0}-l_{1}\right) u\left(\left.x_{2}\right|_{=0}, t\right)\right] .
\end{aligned}
$$

In the static configuration, the effective length of cable segments 1 and 2 is given by the following equation [23]:

$$
l_{j}^{e}=\int_{0}^{l_{j}}\left(\frac{d s}{d x_{j}}\right)^{3} d x_{j}
$$

where $s$ is the arch length of the cable.

By integral operation, the sagging expression for the first cable segment can be written as follows, as shown in Figure 2:

$$
d^{*}=\frac{m g l_{1}^{2} \cos \theta^{*}}{8 H}=\frac{m g l_{1}^{2} \cos (\theta-\alpha)}{8 H} .
$$

Thus,

$$
\begin{aligned}
& l_{1}^{e}=l_{0}\left[\mu_{1}+8 e^{2} \mu_{1}^{3}\left(\frac{1+4 e \mu_{2} \tan \theta}{\sqrt{1+16 e^{2} \mu_{2}^{2}}}\right)^{2}\right], \\
& l_{2}^{e}=l_{0}\left(\mu_{2}+8 e^{2}\left(1-\mu_{1}^{3}\left(\frac{1+4 e \mu_{2} \tan \theta}{\sqrt{1+16 e^{2} \mu_{2}^{2}}}\right)\right),\right.
\end{aligned}
$$

where $\mu_{1}=l_{1} / l_{0}$ is the relative position of transverse forces and $\mu_{2}=l_{2} / l_{0}=1-\mu_{1}$.

From (9) and (12), the following formula is obtained:

$$
\begin{aligned}
& h_{1}=\frac{8 E A e}{l_{1}^{e} l_{0}}\left[\int_{0}^{l_{1}} u\left(x_{1}, t\right) d x_{1}+l_{0}\left(0.5-\mu_{1}\right) u\left(\left.x_{1}\right|_{=l_{1}}, t\right)\right], \\
& h_{2}=\frac{8 E A e}{l_{2}^{e} l_{0}}\left[\int_{0}^{l_{2}} u\left(x_{2}, t\right) d x_{2}-l_{0}\left(0.5-\mu_{1}\right) u\left(\left.x_{2}\right|_{=0}, t\right)\right] .
\end{aligned}
$$

Limited to the case when $j=1,2$, the configuration of the dynamic displacement can be written as follows, using separation of variables:

$$
u\left(x_{j}, t\right)=\varphi\left(x_{j}\right) \mathrm{e}^{\mathrm{i} \omega t},
$$




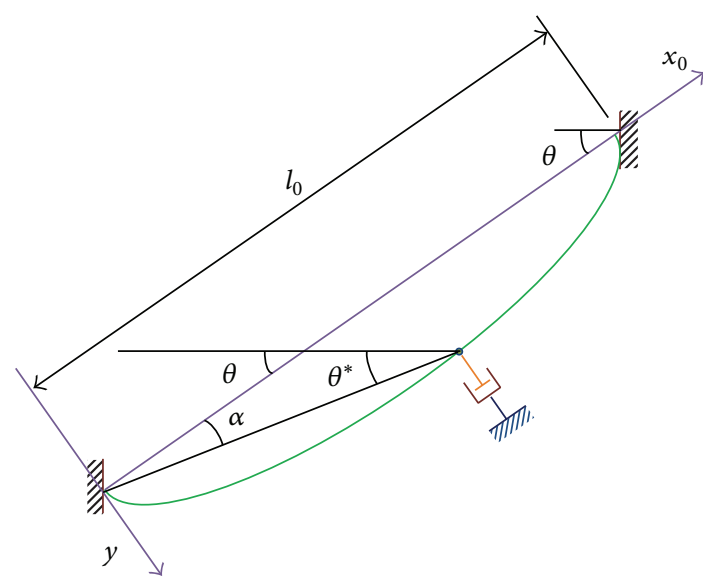

FIGURE 2: Geometry of acted positions of transverse forces.

where $e^{(\cdot)}$ is the exponential function based on natural logarithms, $i=\sqrt{-1}, \omega$ is the angular frequency of vibration, and $\varphi\left(x_{j}\right)$ is the vibration mode function on segment $j$. By substituting (14) into (13), the following formula is obtained:

$$
h_{j}=\widetilde{h}_{j} \mathrm{e}^{\mathrm{i} \omega t},
$$

where

$$
\begin{aligned}
& \tilde{h}_{1}=\frac{8 E A e}{l_{1}{ }^{e} l_{0}}\left[\int_{0}^{l_{1}} \varphi\left(x_{1}\right) d x_{1}+l_{0}\left(0.5-\mu_{1}\right) \varphi\left(\left.x_{1}\right|_{=l_{1}}\right)\right], \\
& \tilde{h}_{2}=\frac{8 E A e}{l_{2}{ }^{e} l_{0}}\left[\int_{0}^{l_{2}} \varphi\left(x_{2}\right) d x_{2}-l_{0}\left(0.5-\mu_{1}\right) \varphi\left(\left.x_{2}\right|_{=0}\right)\right] .
\end{aligned}
$$

It can be observed, by comparing the above equation with the equation given in literature $[17,18]$, that when the effects of transverse forces are considered, the formula for calculating the coefficients of additional cable forces is relevant to both the vibrational mode and the transverse forces.

By substituting (14) into (1), the ordinary differential equation for the vibrational mode function $\varphi\left(x_{j}\right)$ is obtained as follows:

$$
\varphi^{I V}\left(x_{j}\right)-\frac{H}{E I} \varphi^{\prime \prime}\left(x_{j}\right)-\frac{m \omega^{2}}{E I} \varphi\left(x_{j}\right)=-\widetilde{h}_{j} \frac{8 e}{E I l_{0}} .
$$

Let

$$
\begin{aligned}
\xi_{j} & =\frac{x_{j}}{l_{0}}, \\
\widehat{\varphi}\left(\xi_{j}\right) & =\frac{\varphi\left(x_{j}\right) \cdot E I}{\left(m g l_{0}^{4}\right)}, \\
\widehat{h}_{j} & =\frac{\widetilde{h}_{j} \cos \theta}{H} .
\end{aligned}
$$

The dimensionless vibration equation for the two cable segments is listed as follows:

$$
\widehat{\varphi}^{I V}\left(\xi_{j}\right)-\gamma^{2} \widehat{\varphi}^{\prime \prime}\left(\xi_{j}\right)-\widetilde{\omega}^{2} \widehat{\varphi}\left(\xi_{j}\right)=-\widehat{h}_{j},
$$

where $\gamma^{2}=H l_{0}^{2} / E I$ is the ratio of the axial force to bending stiffness of the cable, $\widetilde{\omega}=\omega\left(l_{0}^{2} / \sqrt{E I / m}\right)=\pi^{2}\left(\omega / \omega_{1}^{B}\right)=$ $\pi \gamma\left(\omega / \omega_{1}^{S}\right)$ is the dimensionless frequency of vibration, $\omega_{1}^{s}=$ $\left(\pi / l_{0}\right) \sqrt{H / m}$ is the corresponding fundamental frequency of a taut cable, and $\omega_{1}^{B}=\left(\pi^{2} / l_{0}^{2}\right) \sqrt{E I / m}$ is the corresponding fundamental frequency of a simple beam.

Equation (19) corresponds to the free vibration equation of cable segment $j$, and its corresponding homogeneous equation can be written as

$$
\widehat{\varphi}^{I V}\left(\xi_{j}\right)-\gamma^{2} \widehat{\varphi}^{\prime \prime}\left(\xi_{j}\right)-\widetilde{\omega}^{2} \widehat{\varphi}\left(\xi_{j}\right)=0 .
$$

The general solution of this ordinary differential equation is well solved in references $[18,22]$, which can be written as the following expression

$$
\begin{aligned}
\widehat{\varphi}^{*}\left(\xi_{j}\right)= & A_{1}^{j} \mathrm{e}^{-p \xi_{j}}+A_{2}^{j} \mathrm{e}^{-p\left(\mu_{j}-\xi_{j}\right)} \\
& +A_{3}^{j} \cos \left(q \xi_{j}\right)+A_{4}^{j} \sin \left(q \xi_{j}\right) .
\end{aligned}
$$

The special solution of (19), with the nonhomogeneous item being constant, can be written as

$$
\widehat{\varphi}^{* *}\left(\xi_{j}\right)=\frac{\widehat{h}_{j}}{\widetilde{\omega}^{2}} .
$$

Finally, the complete solution of (19) can be written as

$$
\begin{aligned}
\widehat{\varphi}\left(\xi_{j}\right)= & \widehat{\varphi}^{*}\left(\xi_{j}\right)+\widehat{\varphi}^{* *}\left(\xi_{j}\right) \\
= & A_{1}^{j} \mathrm{e}^{-p \xi_{j}}+A_{2}^{j} \mathrm{e}^{-p\left(\mu_{j}-\xi_{j}\right)}+A_{3}^{j} \cos \left(q \xi_{j}\right) \\
& +A_{4}^{j} \sin \left(q \xi_{j}\right)+\frac{\widehat{h}_{j}}{\widetilde{\omega}^{2}},
\end{aligned}
$$

where

$$
\begin{aligned}
\left.\begin{array}{c}
p \\
q
\end{array}\right\} & =\sqrt{\sqrt{\left(\frac{\gamma^{2}}{2}\right)^{2}+\widetilde{\omega}^{2} \pm \frac{\gamma^{2}}{2}}} \\
& =\sqrt{\sqrt{\left(\frac{H l_{0}^{2}}{2 E I}\right)^{2}+\omega^{2} \frac{m l_{0}^{4}}{E I} \pm \frac{H l_{0}^{2}}{2 E I}}} .
\end{aligned}
$$

In addition, it can be obtained from (24) that

$$
\begin{gathered}
p q=\widetilde{\omega}=\omega \frac{l_{0}^{2}}{\sqrt{E I / m}} \\
p^{2}-q^{2}=\gamma^{2}=\frac{H l_{0}^{2}}{E I} .
\end{gathered}
$$

Equation (23) can be transformed to a matrix form as follows:

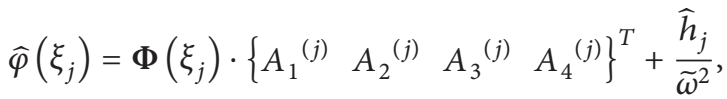


where

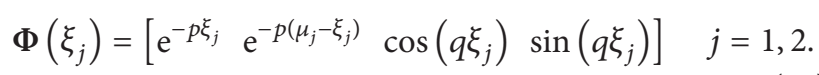

This same method is adopted to make (16) dimensionless, so that

$$
\begin{aligned}
& \widehat{h}_{1}=\eta_{1} \cdot\left[\int_{0}^{\mu_{1}} \widehat{\varphi}\left(\xi_{1}\right) d \xi_{1}+\left(0.5-\mu_{1}\right) \hat{\varphi}\left(\left.\xi_{1}\right|_{=\mu_{1}}\right)\right], \\
& \widehat{h}_{2}=\eta_{2} \cdot\left[\int_{0}^{\mu_{2}} \widehat{\varphi}\left(\xi_{2}\right) d \xi_{2}-\left(0.5-\mu_{1}\right) \hat{\varphi}\left(\left.\xi_{2}\right|_{=0}\right)\right] .
\end{aligned}
$$

In the above equation, $\eta_{j}$ indicates a parameter determined entirely by geometric parameters of the cable and transverse force elements and is given by

$$
\eta_{j}=64 \frac{A l_{0}^{3}}{I l_{j}{ }^{e}} e^{2} .
$$

By substituting (23) into (28), subsequent arrangement, and transposition, a particular solution that is independent of the vibration mode equation is obtained and can be subsequently combined with the general solution to obtain the particular solution of the vibration equation on cable segment $j$, which is shown as follows:

$$
\frac{\widehat{h}_{j}}{\widetilde{\omega}^{2}}=\mathbf{B}^{(j)} \cdot\left\{\begin{array}{lll}
A_{1}{ }^{(j)} & A_{2}{ }^{(j)} \quad A_{3}{ }^{(j)} & \left.A_{4}{ }^{(j)}\right\}^{T}
\end{array}\right.
$$

where matrix $\mathbf{B}^{(j)}$ shows the impact of sagging on the dynamic system, which can be calculated using the following formula:

$$
\mathbf{B}^{(j)}=b_{0}{ }^{(j)} \cdot \begin{cases}\int_{0}^{\mu_{1}} \Phi(\xi) d \xi+\left(0.5-\mu_{1}\right) \Phi\left(\left.\xi_{1}\right|_{=\mu_{1}}\right) & j=1 \\ \int_{0}^{\mu_{2}} \Phi(\xi) d \xi-\left(0.5-\mu_{1}\right) \Phi\left(\left.\xi_{2}\right|_{=0}\right) & j=2 .\end{cases}
$$

When the effects of sagging are considered, the solution for cable segment $j$ can be given in the form of the general solution and the particular solution; namely,

$$
\widehat{\varphi}\left(\xi_{j}\right)=\left(\Phi\left(\xi_{j}\right)+\mathbf{B}^{(j)}\right) \cdot\left\{A_{1}{ }^{(j)} \quad A_{2}{ }^{(j)} \quad A_{3}{ }^{(j)} \quad A_{4}{ }^{(j)}\right\}^{T} .
$$

The amplitude of the additional cable force can be expressed as

$$
\widetilde{h}_{j}=H \widetilde{\omega}^{2} \sec \theta \cdot \mathbf{B}^{(j)} \cdot\left\{A_{1}{ }^{(j)} \quad A_{2}{ }^{(j)} \quad A_{3}{ }^{(j)} A_{4}{ }^{(j)}\right\}^{T} .
$$

\section{Dimensionless Vibrational Mode Function}

The undetermined coefficients in the solutions of the vibrational mode function solution for two cable segments given by formula (32) can be determined through the boundary conditions.
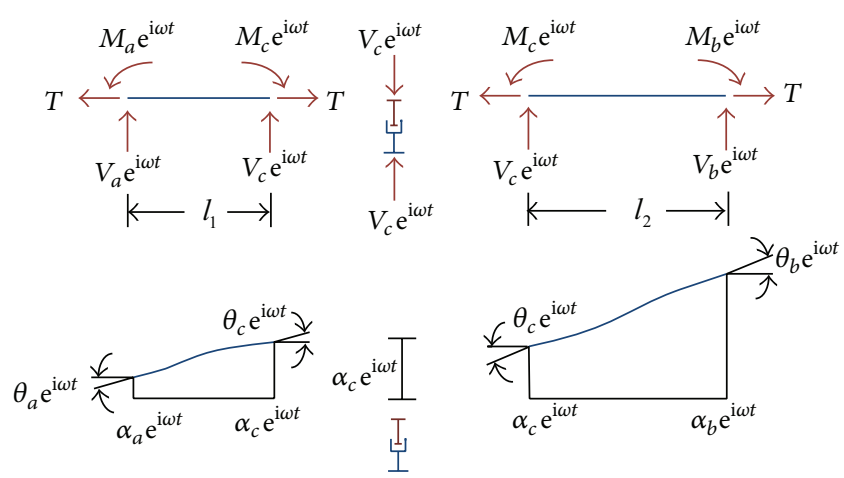

Figure 3: A mechanical model of a discretized cable, damper system.

As shown in Figure 3, the cable system is divided into two independent segments and a transverse force element. The dynamic nodal force and dynamic nodal displacements act on the boundaries of these models. After discretization, the nodal forces for each element include the amplitude of the dynamic nodal shear forces $V_{a}, V_{c}$, and $V_{b}$ and the amplitude of dynamic nodal bending moments $M_{a}, M_{c}$, and $M_{b}$. The nodal displacements include the amplitudes of dynamic displacement $\alpha_{a}, \alpha_{c}$, and $\alpha_{b}$ and the dynamic swing amplitudes $\theta_{a}, \theta_{c}$, and $\theta_{b}$. The time-varying part of these quantities is represented by a complex exponent $\mathrm{e}^{\mathrm{i} \omega t}$, where $\mathrm{i}=\sqrt{-1}$ is the complex unit.

The boundary conditions of the displacement of the two cable segments are given by the following formula:

$$
\begin{aligned}
\alpha_{a} & =\frac{m g l_{0}^{4}}{E I} \widehat{\varphi}\left(\left.\xi_{1}\right|_{=0}\right) \\
\theta_{a} l_{0} & =\frac{m g l_{0}^{4}}{E I} \widehat{\varphi}^{\prime}\left(\left.\xi_{1}\right|_{=0}\right) \\
\alpha_{c} & =\frac{m g l_{0}^{4}}{E I} \widehat{\varphi}\left(\left.\xi_{1}\right|_{=\mu_{1}}\right) \\
\theta_{c} l_{0} & =\frac{m g l_{0}^{4}}{E I} \widehat{\varphi}^{\prime}\left(\left.\xi_{1}\right|_{=\mu_{1}}\right), \\
\alpha_{c} & =\frac{m g l_{0}^{4}}{E I} \widehat{\varphi}\left(\left.\xi_{2}\right|_{=0}\right) \\
\theta_{c} l_{0} & =\frac{m g l_{0}^{4}}{E I} \widehat{\varphi}^{\prime}\left(\left.\xi_{2}\right|_{=0}\right) \\
\alpha_{b} & =\frac{m g l_{0}^{4}}{E I} \widehat{\varphi}\left(\left.\xi_{2}\right|_{=\mu_{2}}\right) \\
\theta_{b} l_{0} & =\frac{m g l_{0}^{4}}{E I} \widehat{\varphi}^{\prime}\left(\left.\xi_{2}\right|_{=\mu_{2}}\right) .
\end{aligned}
$$

For cable segments $j=1,2$, each of the nodal displacements $\left\{\alpha_{a}, \theta_{a} l_{0}, \alpha_{c}, \theta_{c} l_{0}\right\}^{T}$ and $\left\{\alpha_{c}, \theta_{c} l_{0}, \alpha_{b}, \theta_{b} l_{0}\right\}^{T}$ is used as boundary condition, respectively, to obtain the expressions 
for the undetermined coefficients $A_{1}^{(j)} \sim A_{4}^{(j)}$. Equation (32) is substituted into (34) and (35), respectively. Consider

$$
\begin{aligned}
& \widehat{\varphi}\left(\xi_{j}\right)=\varsigma\left(\boldsymbol{\Phi}\left(\xi_{j}\right)+\mathbf{B}^{(j)}\right)\left(\mathbf{C}^{(j)}+\mathbf{I} \cdot \mathbf{B}^{(j)}\right)^{-1}
\end{aligned}
$$

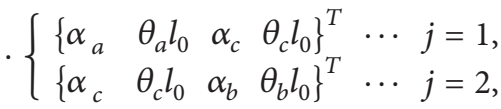

where $\varsigma=E I / m g l_{0}^{4}, \mathbf{I}=\left[\begin{array}{llll}1 & 0 & 1 & 0\end{array}\right]^{T}$, and $\mathbf{C}^{(j)}$ are given by the following equation:

$$
\begin{aligned}
\mathbf{C}^{(j)} & =\left[\begin{array}{llll}
\Phi\left(\left.\xi_{j}\right|_{=0}\right) & \Phi^{\prime}\left(\left.\xi_{j}\right|_{=0}\right) & \boldsymbol{\Phi}\left(\left.\xi_{j}\right|_{=\mu_{j}}\right) & \boldsymbol{\Phi}^{\prime}\left(\left.\xi_{j}\right|_{=\mu_{j}}\right)
\end{array}\right]^{T} \\
& =\left[\begin{array}{cccc}
1 & \mathrm{e}^{-p \mu_{j}} & 1 & 0 \\
-p & p \mathrm{e}^{-p \mu_{j}} & 0 & q \\
\mathrm{e}^{-p \mu_{j}} & 1 & \cos \left(q \mu_{j}\right) & \sin \left(q \mu_{j}\right) \\
-p \mathrm{e}^{-p \mu_{j}} & p & -q \sin \left(q \mu_{j}\right) & q \cos \left(q \mu_{j}\right)
\end{array}\right] .
\end{aligned}
$$

\section{Dynamic Stiffness Matrix of} the Cable Damper System

All internal forces on cable segment $j$ can be expressed as follows:

$$
\begin{aligned}
V\left(x_{j}, t\right) & =\left(E I \frac{d^{3} \varphi}{d x_{j}{ }^{3}}-H \frac{d \varphi}{d x_{j}}\right) \cdot \mathrm{e}^{\mathrm{i} \omega t} \\
& =m g l_{0}\left(\widehat{\varphi}^{\prime \prime \prime}\left(\xi_{j}\right)-\gamma^{2} \widehat{\varphi}^{\prime}\left(\xi_{j}\right)\right) \cdot \mathrm{e}^{\mathrm{i} \omega t} \\
M\left(x_{j}, t\right) & =E I \frac{d^{2} \varphi}{d x_{j}^{2}} \mathrm{e}^{\mathrm{i} \omega t}=m g l_{0}^{2} \widehat{\varphi}^{\prime \prime}\left(\xi_{j}\right) \mathrm{e}^{\mathrm{i} \omega t} .
\end{aligned}
$$

The equilibrium conditions for the two cable segments are given by the following set of equations:

$$
\begin{array}{cc}
V\left(\left.x_{1}\right|_{=0}, t\right)=V_{a} \mathrm{e}^{\mathrm{i} \omega t}, & -M\left(\left.x_{1}\right|_{=0}, t\right)=M_{a} \mathrm{e}^{\mathrm{i} \omega t} \\
-V\left(\left.x_{1}\right|_{=l_{1}}, t\right)=V_{c} \mathrm{e}^{\mathrm{i} \omega t}, & M\left(\left.x_{1}\right|_{=l_{1}}, t\right)=M_{c} \mathrm{e}^{\mathrm{i} \omega t} \\
\vdots & \vdots \\
V\left(\left.x_{2}\right|_{=0}, t\right)=V_{c} \mathrm{e}^{\mathrm{i} \omega t}, & -M\left(\left.x_{2}\right|_{=0}, t\right)=M_{c} \mathrm{e}^{\mathrm{i} \omega t} \\
-V\left(\left.x_{2}\right|_{=l_{2}}, t\right)=V_{b} \mathrm{e}^{\mathrm{i} \omega t}, & M\left(\left.x_{2}\right|_{=l_{2}}, t\right)=M_{b} \mathrm{e}^{\mathrm{i} \omega t} .
\end{array}
$$

Equation (39) is transformed to matrix form according to the expressions for cable segments. Consider

$$
\begin{aligned}
& \left\{\begin{array}{c}
V_{a} \\
\frac{M_{a}}{l_{0}} \\
V_{c} \\
\frac{M_{c}}{l_{0}}
\end{array}\right\}=m g l_{0}\left[\begin{array}{c}
\widehat{\varphi}^{\prime \prime \prime}\left(\left.\xi_{1}\right|_{=0}\right)-\gamma^{2} \widehat{\varphi}^{\prime}\left(\left.\xi_{1}\right|_{=0}\right) \\
-\widehat{\varphi}^{\prime \prime}\left(\left.\xi_{1}\right|_{=0}\right) \\
-\widehat{\varphi}^{\prime \prime \prime}\left(\left.\xi_{1}\right|_{=\mu_{1}}\right)+\gamma^{2} \widehat{\varphi}^{\prime}\left(\left.\xi_{1}\right|_{=\mu_{1}}\right) \\
\widehat{\varphi}^{\prime \prime}\left(\left.\xi_{1}\right|_{=\mu_{1}}\right)
\end{array}\right] \\
& =\frac{E I}{l_{0}^{3}} \mathbf{D}^{(1)} \cdot\left(\mathbf{C}^{(1)}+\mathbf{I} \cdot \mathbf{B}^{(1)}\right)^{-1} \\
& \cdot\left\{\begin{array}{llll}
\alpha_{a} & \theta_{a} l_{0} & \alpha_{c} & \theta_{c} l_{0}
\end{array}\right\}^{T}, \\
& \left\{\begin{array}{c}
V_{c} \\
\frac{M_{c}}{l_{0}} \\
V_{b} \\
\frac{M_{b}}{l_{0}}
\end{array}\right\}=m g l_{0}\left[\begin{array}{c}
\hat{\varphi}^{\prime \prime \prime}\left(\left.\xi_{2}\right|_{=0}\right)-\gamma^{2} \widehat{\varphi}^{\prime}\left(\left.\xi_{2}\right|_{=0}\right) \\
-\widehat{\varphi}^{\prime \prime}\left(\left.\xi_{2}\right|_{=0}\right) \\
-\widehat{\varphi}^{\prime \prime \prime}\left(\left.\xi_{2}\right|_{=\mu_{2}}\right)+\gamma^{2} \widehat{\varphi}^{\prime}\left(\left.\xi_{2}\right|_{=\mu_{2}}\right) \\
\hat{\varphi}^{\prime \prime}\left(\left.\xi_{2}\right|_{=\mu_{2}}\right)
\end{array}\right] \\
& =\frac{E I}{l_{0}^{3}} \mathbf{D}^{(2)} \cdot\left(\mathbf{C}^{(2)}+\mathbf{I} \cdot \mathbf{B}^{(2)}\right)^{-1} \\
& \cdot\left\{\begin{array}{llll}
\alpha_{c} & \theta_{c} l_{0} & \alpha_{b} & \theta_{b} l_{0}
\end{array}\right\}^{T},
\end{aligned}
$$

where the matrix $\mathbf{D}^{(j)}$ is defined as follows:

$$
\mathbf{D}^{(j)}=\left[\begin{array}{c}
\left(\Phi^{\prime \prime \prime}\left(\left.\xi_{j}\right|_{=0}\right)\right)-\gamma^{2}\left(\Phi^{\prime}\left(\left.\xi_{j}\right|_{=0}\right)\right) \\
-\left(\Phi^{\prime \prime}\left(\left.\xi_{j}\right|_{=0}\right)\right) \\
-\left(\boldsymbol{\Phi}^{\prime \prime \prime}\left(\left.\xi_{j}\right|_{=\mu_{j}}\right)\right)+\gamma^{2}\left(\boldsymbol{\Phi}^{\prime}\left(\left.\xi_{j}\right|_{=\mu_{j}}\right)\right) \\
\left(\boldsymbol{\Phi}^{\prime \prime}\left(\left.\xi_{j}\right|_{=\mu_{j}}\right)\right)
\end{array}\right]
$$

The dynamic stiffness matrix of the cable segment is defined as follows:

$$
\begin{aligned}
\mathbf{K}^{(j)} & =m g l_{0} \varsigma \cdot \mathbf{D}^{(j)} \cdot\left(\mathbf{C}^{(j)}+\mathbf{I} \cdot \mathbf{B}^{(j)}\right)^{-1} \\
& =\frac{E I}{l_{0}{ }^{3}} \mathbf{D}^{(j)} \cdot\left(\mathbf{C}^{(j)}+\mathbf{I} \cdot \mathbf{B}^{(j)}\right)^{-1}
\end{aligned}
$$


For each cable segment, the relationship between the dynamic nodal force and the dynamic displacement is written in matrix form as follows:

$$
\begin{gathered}
\mathbf{K}^{(1)}\left\{\begin{array}{c}
\alpha_{a} \\
\theta_{a} l_{0} \\
\alpha_{c} \\
\theta_{c} l_{0}
\end{array}\right\}=\left\{\begin{array}{c}
V_{a} \\
\frac{M_{a}}{l_{0}} \\
V_{c} \\
M_{c} \\
l_{0}
\end{array}\right\}, \\
\mathbf{K}^{(2)}\left\{\begin{array}{c}
\alpha_{c} \\
\theta_{c} l_{0} \\
\alpha_{b} \\
\theta_{b} l_{0}
\end{array}\right\}=\left\{\begin{array}{c}
V_{c} \\
\frac{M_{c}}{l_{0}} \\
V_{b} \\
M_{b} \\
l_{0}
\end{array}\right\} .
\end{gathered}
$$

After combining the two equations, the equation for the relationship between the total nodal force and the total displacement is established. Furthermore, the dynamic equilibrium equation for a cable acted upon by transverse forces, with clamped boundary conditions at both ends, is obtained:

$$
\mathbf{K}^{(0)}\left\{\begin{array}{c}
\alpha_{c} \\
\theta_{c} l_{0}
\end{array}\right\}=\left\{\begin{array}{c}
V_{c} \\
M_{c} \\
\hline l_{0}
\end{array}\right\},
$$

where the total dynamic stiffness matrix $\mathbf{K}^{(0)}$ of the cable system with two degrees of freedom can be obtained by combining $\mathbf{K}^{(1)}$ and $\mathbf{K}^{(2)}$. The assembly process can be written in matrix operator form as follows:

$$
\mathbf{K}^{(0)}=\mathbf{I}_{1} \cdot \mathbf{K}^{(1)} \cdot \mathbf{I}_{1}{ }^{T}+\mathbf{I}_{2} \cdot \mathbf{K}^{(2)} \cdot \mathbf{I}_{2}{ }^{T} .
$$

The matrix operator in the equation is as follows:

$$
\mathbf{I}_{1}=\left[\begin{array}{llll}
0 & 0 & 1 & 0 \\
0 & 0 & 0 & 1
\end{array}\right], \quad \mathbf{I}_{2}=\left[\begin{array}{llll}
1 & 0 & 0 & 0 \\
0 & 1 & 0 & 0
\end{array}\right] \mathbf{K}^{(0)}
$$

$\mathbf{K}^{(0)}$ can be written in the form of matrix elements as follows:

$$
\mathbf{K}^{(0)}=\frac{E I}{l_{0}^{3}}\left[\begin{array}{ll}
k_{11}^{(0)} & k_{12}^{(0)} \\
k_{21}^{(0)} & k_{22}^{(0)}
\end{array}\right] \text {, }
$$

where $k_{11}^{(0)}=k_{33}^{(1)}+k_{11}^{(2)}, k_{12}^{(0)}=k_{34}^{(1)}+k_{12}^{(2)} ; k_{21}^{(0)}=k_{43}^{(1)}+k_{21}^{(2)}$, $k_{22}^{(0)}=k_{44}^{(1)}+k_{22}^{(2)} ; k_{33}^{(1)} ; k_{44}^{(1)}, k_{43}^{(1)}$, and $k_{34}^{(1)}$ and $k_{11}^{(2)}, k_{22}^{(2)}, k_{21}^{(2)}$, and $k_{12}^{(2)}$ corresponds to $\mathbf{K}^{(j)}$, respectively.

It can be seen from (44) and its derivation that the equilibrium equation takes into account the impact of bending stiffness, clamped boundary conditions, and cable sagging. The factors considered here comprise a model that is most closely related to the cables used in actual projects. Therefore, in theory, this equation is able to describe more accurately the cable damper system in actual projects.

Similarly, by letting the above matrix $\mathbf{B}^{(j)}$ be zero, the dynamic stiffness of each cable segment, not considering sagging, is obtained as follows:

$$
\mathbf{K}^{(j)}=\frac{E I}{l_{0}^{3}} \mathbf{D}^{(j)} \cdot\left(\mathbf{C}^{(j)}\right)^{-1} .
$$

The overall dynamic stiffness matrix of the cable can be obtained using (45). The dynamic equilibrium equation of the cable can be obtained by using (44).

The horizontal dynamic stiffness of the cable with a single degree of freedom can be obtained by rearranging (47) to the following form:

$$
k_{\mathrm{dyn}}=k_{11}^{(0)}-\frac{k_{12}^{(0)} \cdot k_{21}^{(0)}}{k_{22}^{(0)}} .
$$

\section{Verification}

To verify the accuracy of the two analytical methods for lateral dynamic stiffness, a finite element method is used to model the cable, and then a model reduction method is used to obtain the condensed stiffness and condensed mass corresponding to the degree of freedom. At this point, the corresponding transverse dynamic stiffness of cables is obtained.

Corresponding to the analytical method presented in this paper, the FEM tool-box, calfem-3.4, which is running at Matlab environment, is used to establish the finite element model. The cable and the damper are modeled by the two-dimensional plane beam element and a linear viscous damper model, respectively. The FEM model takes into account the effects of bending stiffness, inclination angle, clamped boundary conditions, and sagging, as well as the contributions of the cable forces and bending moments of the geometric stiffness matrix of cable. Reduction of mass and stiffness is conducted, for the two corresponding degrees of freedom, on the element acted upon by transverse forces using Guyan's static condensation. Linear interpolation is then used to obtain the transverse dynamic stiffness matrix for the two degrees of freedom of the subordinate target point.

The basic parameters of the cable with both ends clamped which is used in the study are shown in Table 1.

\subsection{Transverse Dynamic Stiffness Matrix of Two Degrees of} Freedom. According to the deduction process in the last section, the corresponding transverse static stiffness of the cable can be obtained by merely letting the circular frequency be $\omega=0$. At this point, the dynamic stiffness at the estimated value of the modal frequency of the cable (provided by the formula of taut string theory in (50)) is investigated, which has a vital significance for the actual project.

$$
\omega=\frac{k \pi}{l_{0}} \sqrt{\frac{H}{m}} .
$$


TABLE 1: Basic parameters of the cable.

\begin{tabular}{lccc}
\hline Modulus of elasticity & Moment of inertia & Mass per unit length & Area \\
\hline $2 \times 10^{11} \mathrm{~Pa}$ & $8.004 \times 10^{-6} \mathrm{~m}^{4}$ & $96.85 \mathrm{~kg} / \mathrm{m}$ & $1.24 \times 10^{-2} \mathrm{~m}^{2}$ \\
Chord-wise force & Length & Relative location of transverse force & Inclined angle \\
$3 \times 10^{6} \mathrm{~N}$ & $200 \mathrm{~m}$ & 0.5 & $30^{\circ}$ \\
\hline
\end{tabular}

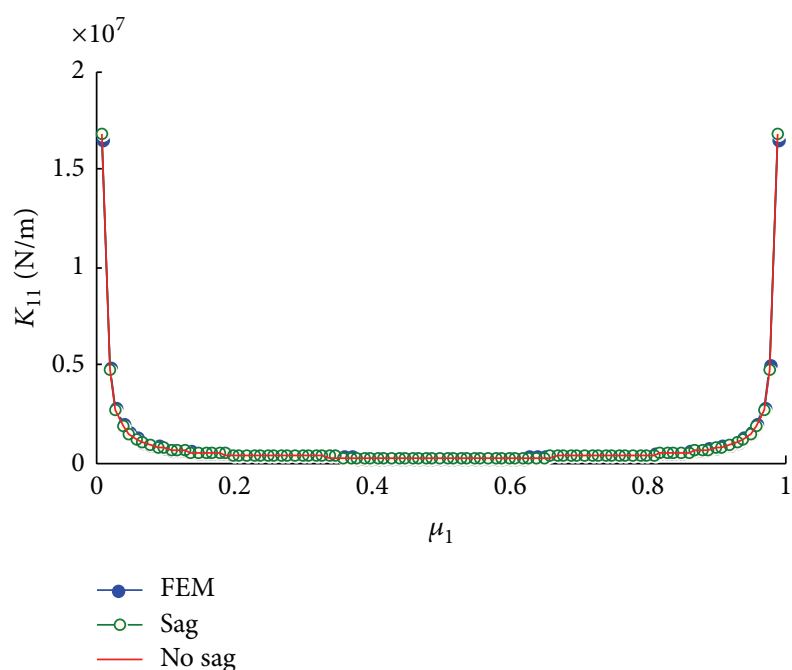

(a)

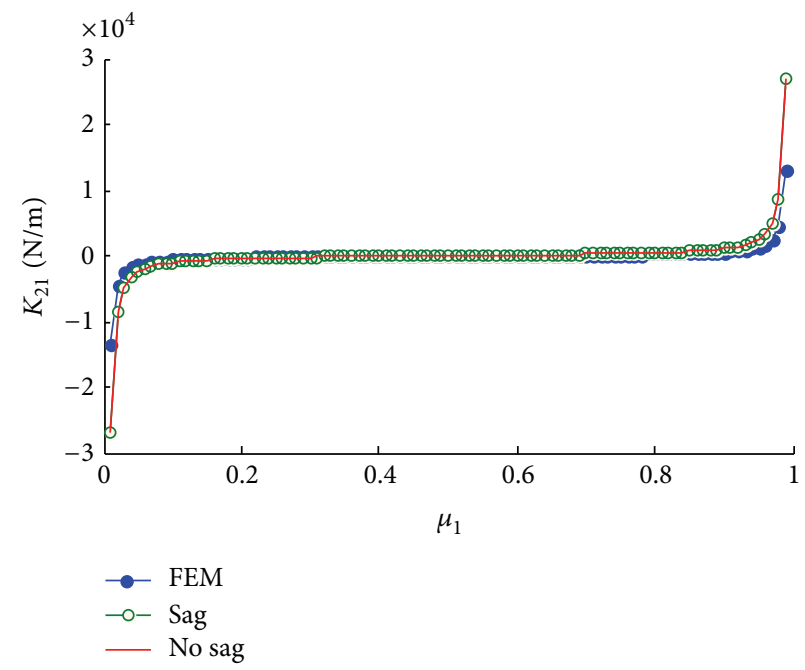

(c)

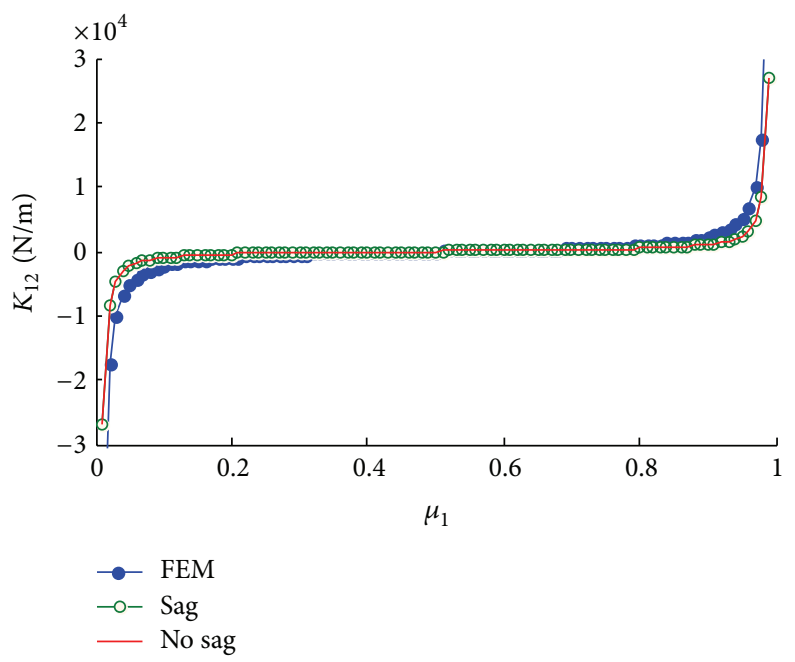

(b)

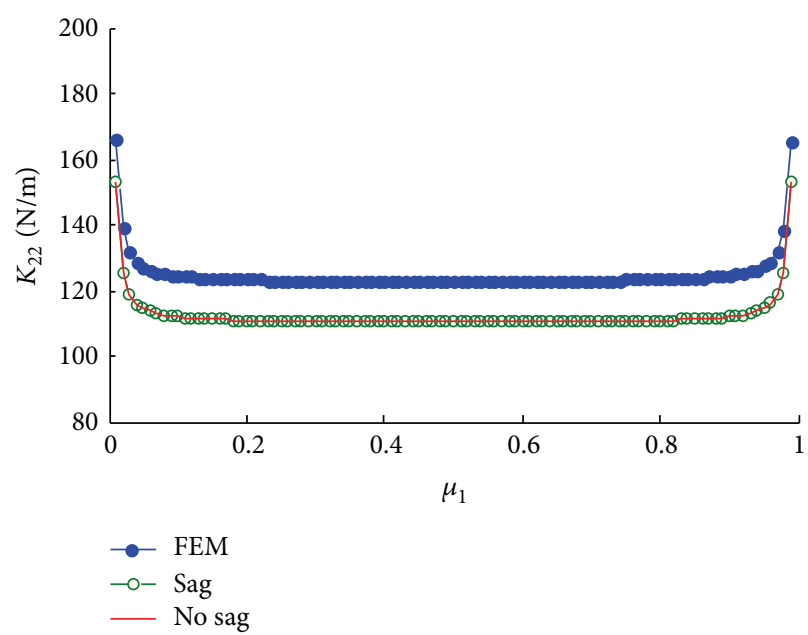

(d)

FIGURE 4: Comparison of the terms of the static stiffness matrix along the length of cable with two transverse degrees of freedom using the three presented methods $(\omega=0$, unit: $\mathrm{N} / \mathrm{m})$.

Figure 4 shows the variations in static stiffness along the cable length using the three methods. The transverse axis $\mu_{1}$ represents the relative chord-wise length from the position of the transverse force to the anchor point of the inclined cable. The vertical axis represents the stiffness term. Due to the treatment of nodal displacements and nodal forces as described in the above section, the four terms have the same dimension. Table 2 shows a comparison of the values of the stiffness terms at the position when $\mu_{1}$ is $0.05,0.1,0.25,0.5$,
$0.75,0.9$, and 0.95 , respectively. Comparison of the four terms at the midpoint of the span is carried out.

As can be seen in Figure 4, the distributions of various terms of the two-dimensional dynamic stiffness matrix have similar patterns, in that the stiffness coefficient of a cable along the main diagonal of the matrix is extremely large at both ends of the cable. Moving the application point of the transverse force toward the midpoint of the cable causes this coefficient to decline rapidly. However, when $\mu_{1}$ varies within 
TABLE 2: Comparison of values of dynamic stiffness at different locations.

\begin{tabular}{lccccccc}
\hline Method $^{1}$ & 0.05 & 0.1 & 0.25 & 0.5 & 0.75 & 0.9 & 0.95 \\
\hline One $\left(K_{11}\right)^{2}$ & $1.4807 \times 10^{6}$ & $7.2566 \times 10^{5}$ & $3.3728 \times 10^{5}$ & $2.4966 \times 10^{5}$ & $3.3100 \times 10^{5}$ & $7.1567 \times 10^{5}$ & $1.4695 \times 10^{6}$ \\
Two $\left(K_{11}\right)$ & $1.4690 \times 10^{6}$ & $7.1449 \times 10^{5}$ & $3.2801 \times 10^{5}$ & $2.4356 \times 10^{5}$ & $3.2801 \times 10^{5}$ & $7.1449 \times 10^{5}$ & $1.4690 \times 10^{6}$ \\
Three $\left(K_{11}\right)$ & $1.5323 \times 10^{6}$ & $7.4813 \times 10^{5}$ & $3.4502 \times 10^{5}$ & $2.5732 \times 10^{5}$ & $3.4728 \times 10^{5}$ & $7.5560 \times 10^{5}$ & $1.5478 \times 10^{6}$ \\
Relative error ${ }^{3}(\%)$ & 3.3659 & 3.0044 & 2.2433 & 2.9752 & 4.6871 & 5.2844 & 5.0586 \\
One $\left(K_{22}\right)^{2}$ & 114.47 & 111.96 & 110.67 & 110.40 & 110.69 & 111.99 & 114.50 \\
Two $\left(K_{22}\right)$ & 114.47 & 111.95 & 110.66 & 110.38 & 110.66 & 111.95 & 114.47 \\
Three $\left(K_{22}\right)$ & 127.29 & 124.55 & 123.18 & 122.93 & 123.28 & 124.70 & 127.39 \\
Relative error $(\%)$ & 10.07 & 10.11 & 10.15 & 10.19 & 10.21 & 10.20 & 10.11 \\
\hline
\end{tabular}

Note: ${ }^{1}$ method one is the analytic method considering sagging effects, which corresponds to (42). Method two is the analytic method without considering sagging effects, which corresponds to (48). Method three is the finite element method. ${ }^{2} K_{11}$ and $K_{22}$ are the first and second terms on the main diagonal of matrix, respectively. ${ }^{3}$ The relative error indicates the difference between the results obtained via method one and method three.

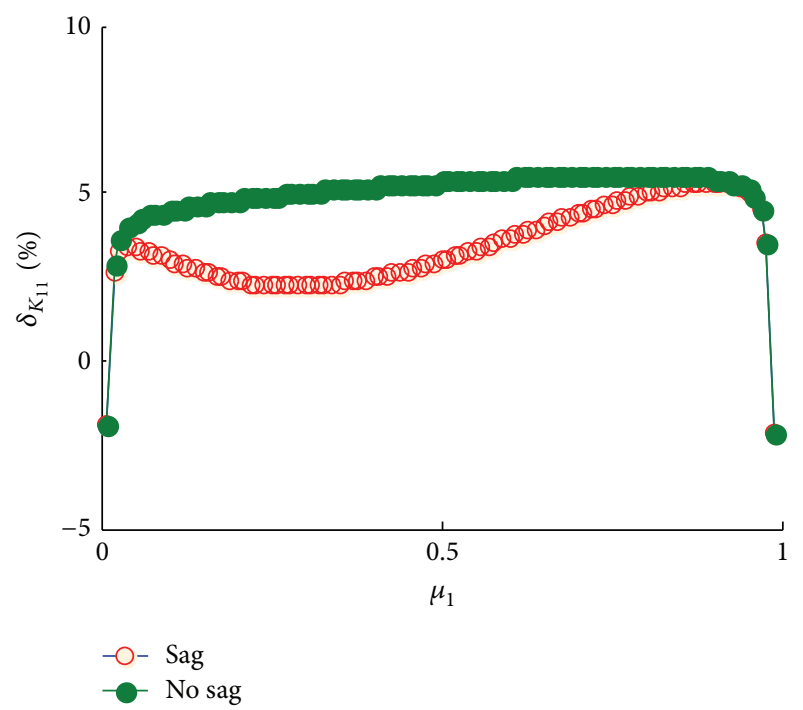

(a)

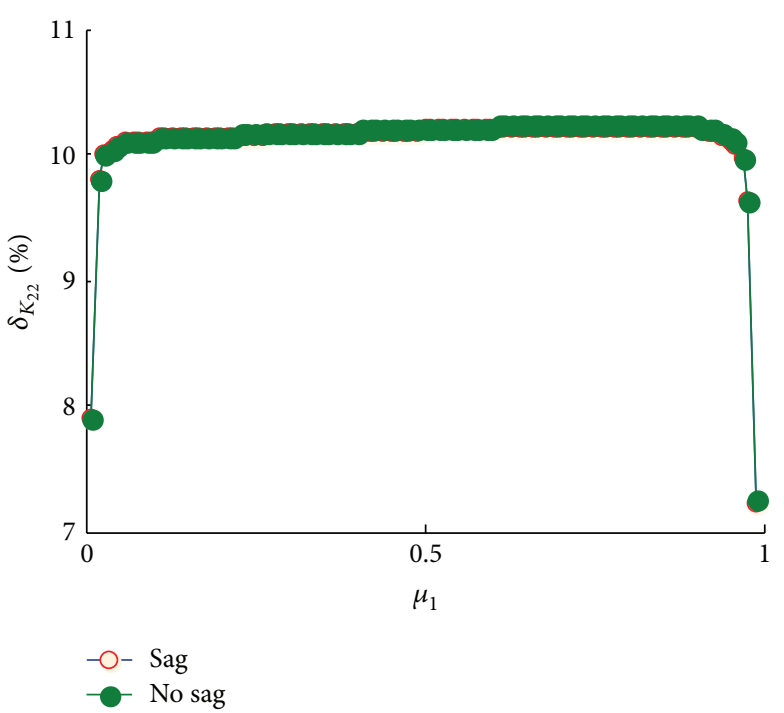

(b)

FIGURE 5: Relative error between main diagonal terms and the finite element method.

$[0.1,0.9]$, it does so smoothly, in the shape of a pan. The secondary diagonal terms transform rapidly from negative infinity to the smooth range in the middle of cable and then to positive infinity along the cable length.

The results obtained via the three presented methods for calculating the transverse static stiffness of the cable are consistent. The relationship between the values obtained in each method can be evaluated according to the following definition of error:

$$
\begin{gathered}
\delta_{k_{j j}}{ }^{\text {Sag }}=100 \times \frac{k_{j j}{ }^{0, \mathrm{FEM}}-k_{j j}{ }^{0, \mathrm{Sag}}}{k_{j j}{ }^{0, \mathrm{FEM}}} \\
\delta_{k_{j j}{ }^{\mathrm{noSag}}}=100 \times \frac{k_{j j}{ }^{0, \mathrm{FEM}}-k_{j j}{ }^{0, \mathrm{noSag}}}{k_{j j}{ }^{0, \mathrm{FEM}}},
\end{gathered}
$$

where the subscript is $j i=11,22$. The superscripts 0 , FEM, 0 , Sag, and 0 , noSag represent the total stiffness terms obtained using the finite element method, by considering and neglecting sagging effects, respectively. As can be seen from Table 2 and Figure 5, the numerical differences between the stiffness terms obtained via the three presented methods are very small. Along the cable length, the error between the results of the first term on the main diagonal $k_{11}^{(0)}$ and the finite element method is less than $5.2 \% ; k_{22}^{(0)}$ is also small, within $11 \%$. Though the relative error is given by Guyan's static condensation using finite element analysis, the rigidity of the dynamic condensation itself also has errors. However, since the error of static stiffness is very small when $\omega=0$, this case can be used as a reference of accuracy in the study. It is noted that, in this study, the obtained variation pattern of the transverse static stiffness of the cables along the cable length is reliable and has good computational accuracy. The dynamic stiffness method, taking sagging effects into account, is even closer to the static condensation results using finite element analysis. 


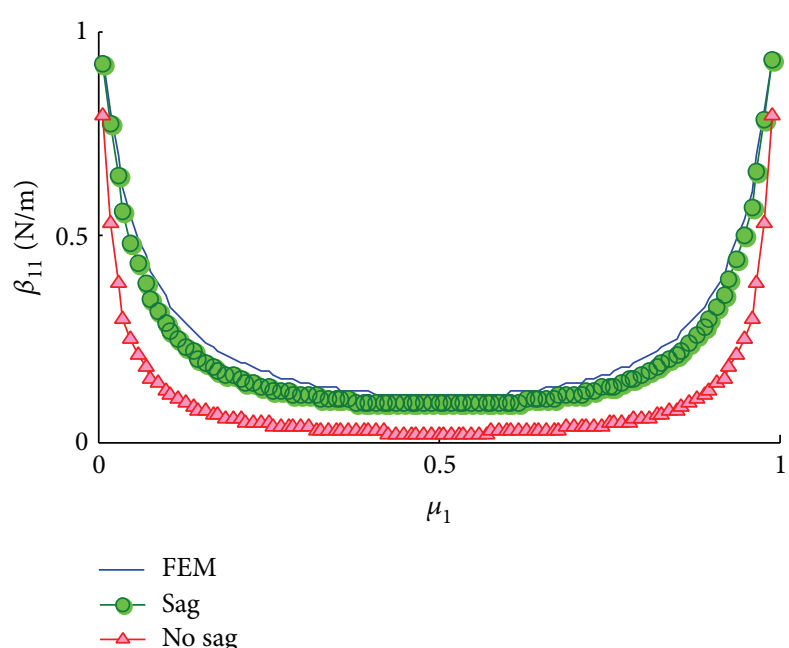

(a)

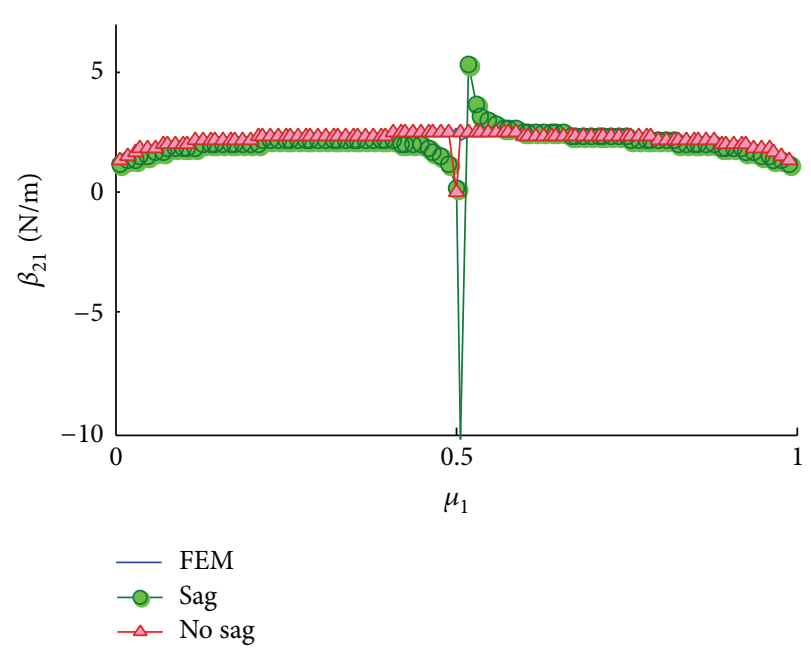

(c)

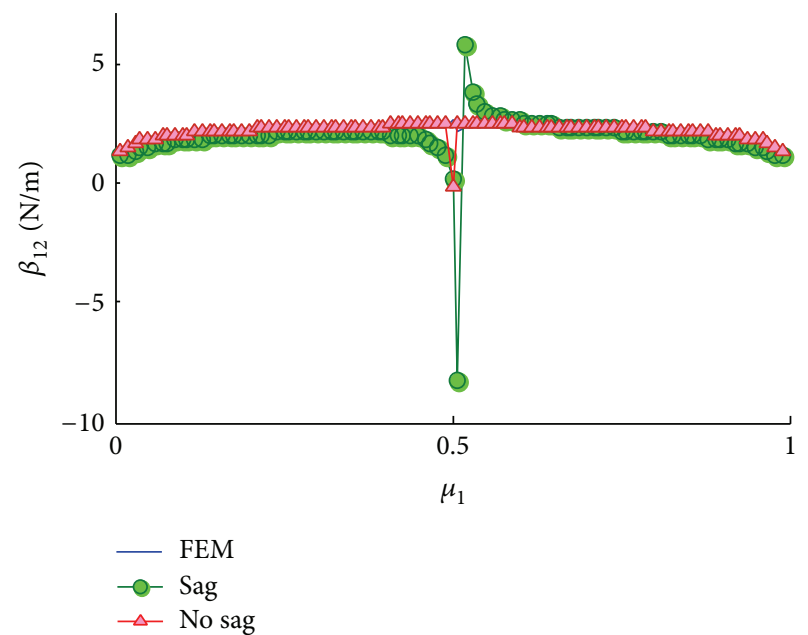

(b)

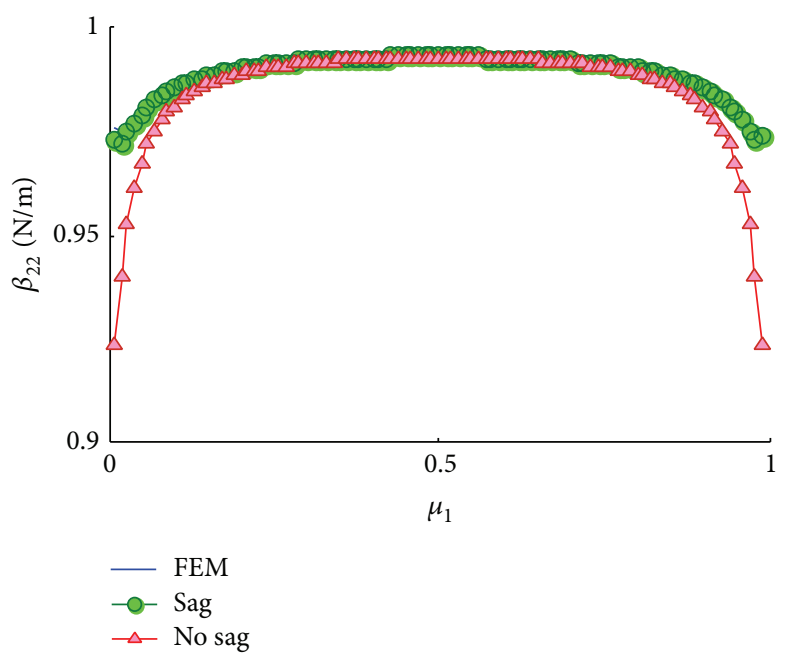

(d)

FiguRE 6: Comparison between the coefficient of the first-order dynamic stiffness obtained using the three presented methods $\left(\omega_{1}^{s}=2.765\right)$.

Before analyzing the dynamic stiffness obtained using the three presented methods, the coefficient of dynamic stiffness $\beta_{i j}$ is defined as follows:

$$
\beta_{i j}=\frac{K_{i j}{ }^{0}\left(\omega_{1}^{s}\right)}{K_{i j}{ }^{0}(0)},
$$

where $\omega_{1}^{s}$ is the estimated value of the first-order modal frequency of the cable according to the taut string equation, $K_{i j}{ }^{0}(\cdot)$ indicates the dynamic stiffness for a certain frequency, and $K_{i j}{ }^{0}(0)$ represents the static stiffness.

Figure 6 shows the variations of the dynamic stiffness of the cable along its length, based on the estimated value of the first-order modal frequency using the three presented methods. The coefficient of the dynamic stiffness of $\beta_{11}$ corresponding to $k_{11}^{(0)}$ shows a distribution along the cable length with a shape of a positively laid pan, where the midpoint of the cable is a minimum, having a value of approximately $10 \%$ of the static stiffness. Conversely, $\beta_{22}$ shows a distribution with a shape of an inverted pan, where the midpoint of cable has a maximum value, getting closer to the static stiffness as it moves from the ends to the midpoint. The nearer one is to the center point, the closer the value is to the static stiffness. $\beta_{12}$ and $\beta_{21}$, which correspond to the secondary diagonal, show discontinuities at the midpoint of cable. Their values approach positive and negative infinity, respectively.

The results of a comparison of the three presented methods can be drawn from Figures 6(a) and 6(b). The dynamic stiffness obtained using the method that considers sagging and obtained by the condensation method using finite element analysis yields results that are close to each other, whereas the method that does not take sagging effects into account produces results that are quite different. This shows that sagging effects have a significant impact on the dynamic stiffness of the cable near the first-order vibrational frequency and thus should be considered in calculations. 


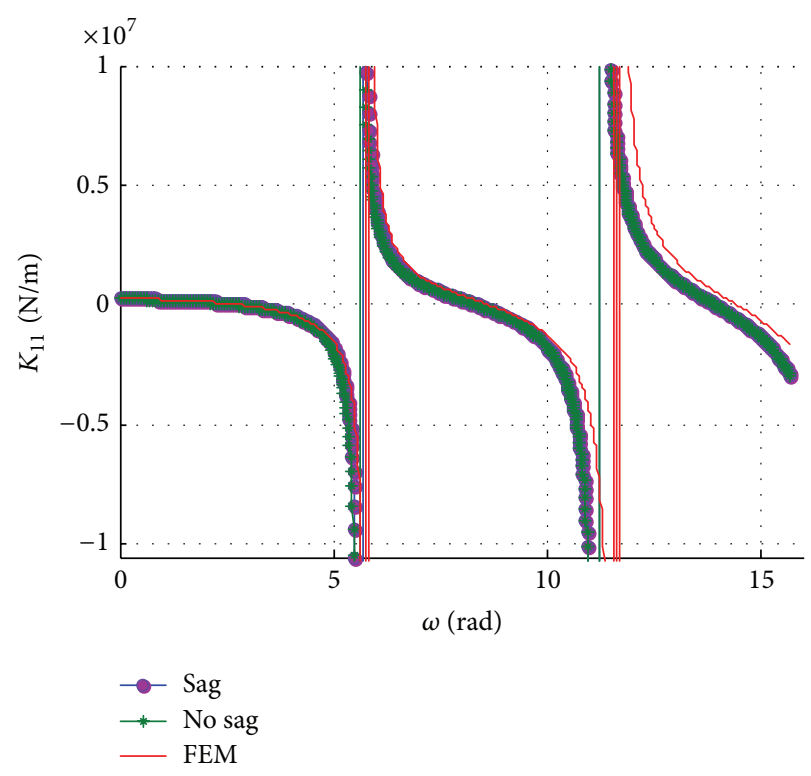

(a)

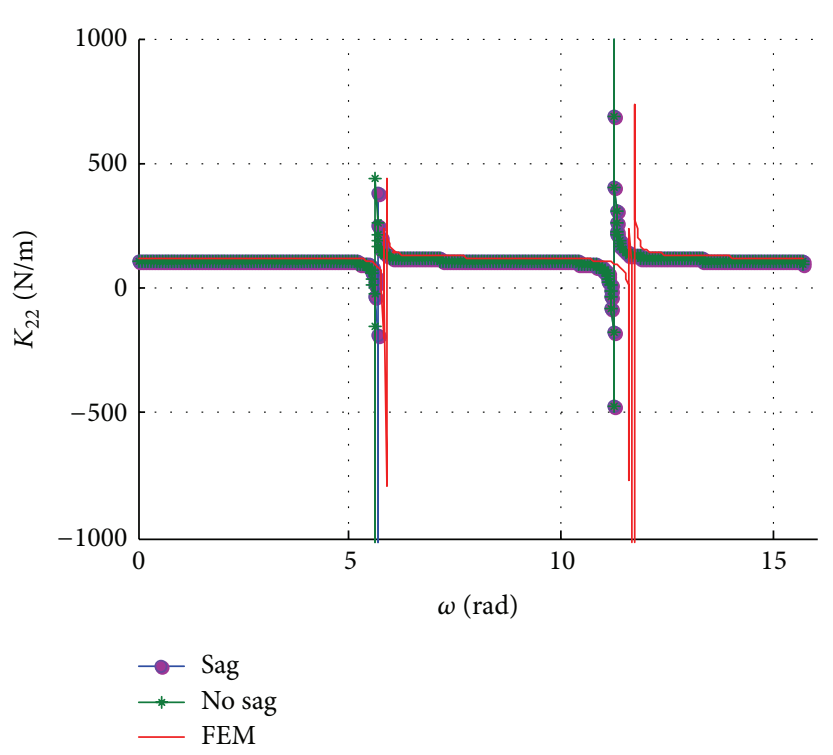

(b)

FIGURE 7: Variation curve for the frequency domain of the dynamic stiffness at the midpoint of a cable $\left(\mu_{1}=0.5\right)$.

In some excitation tests of cables, the excitation points of even-order modes are often chosen to be at the midpoints of the cables. Therefore, it is crucial to consider the dynamic stiffness of the cables at the midpoint in engineering vibration tests. Figures $7(\mathrm{a})$ and $7(\mathrm{~b})$ show the variation curve of the main diagonal terms of the dynamic stiffness matrix at the midpoint of a cable within the frequency domain (i.e., within $[0,5 \pi])$. As can be seen from the figure, the dynamic stiffness at the midpoint of the cable has an infinite discontinuity for even order. There is a zero crossing point in each continuous interval, namely, the odd-order modal frequency. The variation curves of the dynamic stiffness, with frequencies obtained using the three presented methods, are basically consistent. This shows that the calculated dynamic stiffness using the three methods is basically consistent within the investigated frequency range, with very small numerical differences. Figures 6 and 7 show that the calculated results given in this work for the dynamic stiffness at any frequency, using the analytical algorithm for dynamic stiffness, while considering sagging effects, are accurate and reliable.

\subsection{Transverse Dynamic Stiffness for a Single Degree of Free-} dom. The same conclusion can also be drawn by comparing the transverse dynamic stiffness of the cable for a single degree of freedom using different methods. Figure 8 shows the variations of the static stiffness along the cable length for a single degree of freedom using three methods. Figures 9(a)9(d) show the variations of the coefficients of the dynamic stiffness along the cable length, based on the estimated value of the first-, second-, third-, and fourth-order modal frequencies, respectively (with a reference static stiffness value obtained via a finite element method). As can be seen from Figures 8 and 9, the analytical solution of the dynamic

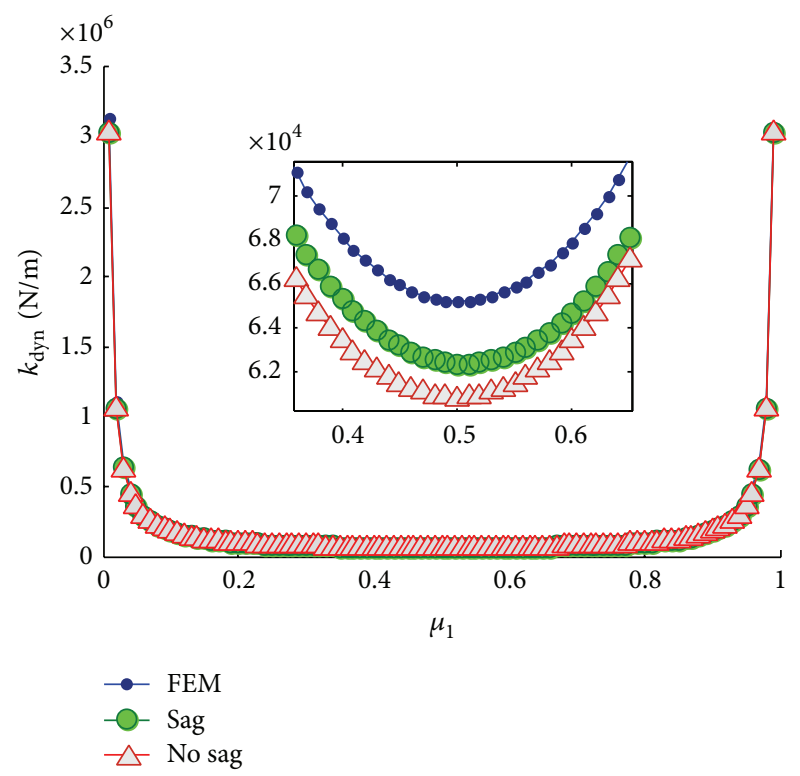

Figure 8: A comparison between the static stiffness of cables with a single transverse degree of freedom using different methods $(\omega=0)$.

stiffness is accurate when considering sagging effects. The analytical solution when sagging effects are neglected is also accurate for calculating static stiffness, but a large error occurs when using this solution to calculate the first-order dynamic stiffness. The static stiffness obtained by Guyan's static condensation using a finite element method gives results that are very close to those of the former two methods. The accuracy of the Guyan approach is acceptable for calculating the dynamic stiffness at fundamental frequencies. However, as the order increases, the results of the Guyan approach 


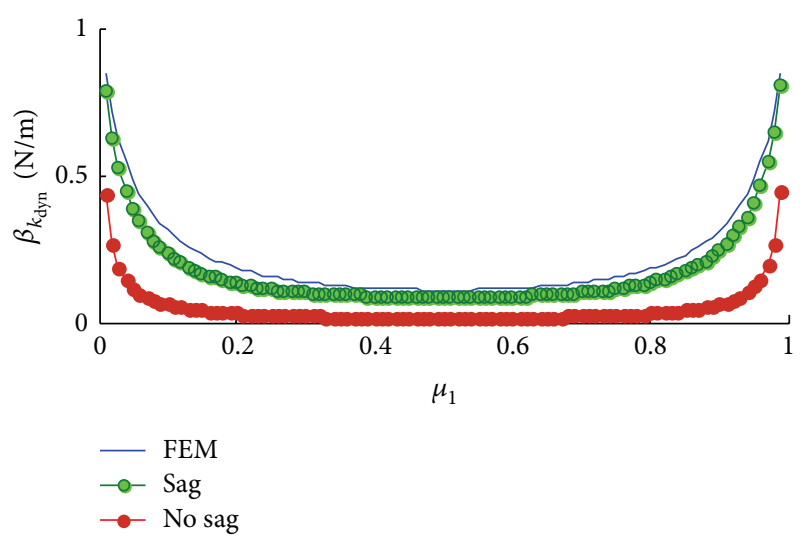

(a) Mode 1

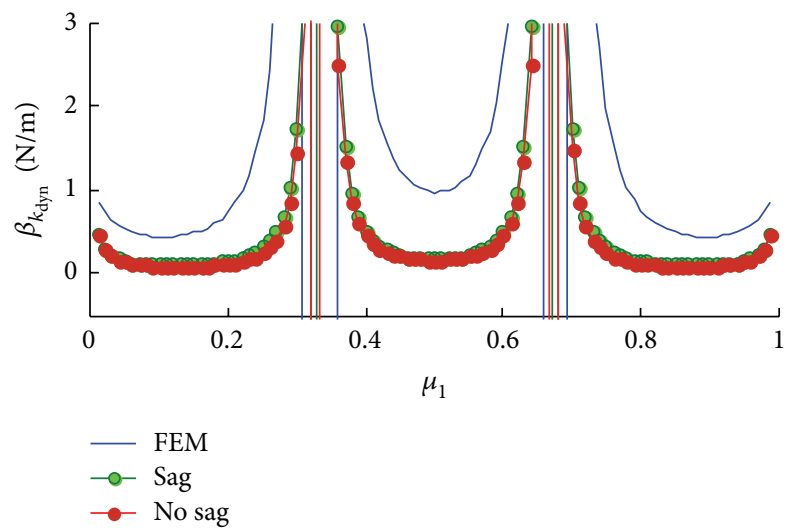

(c) Mode 3

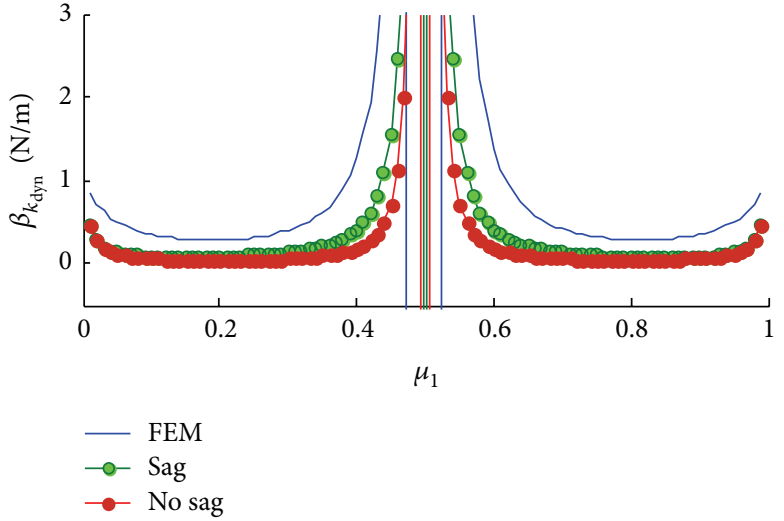

(b) Mode 2

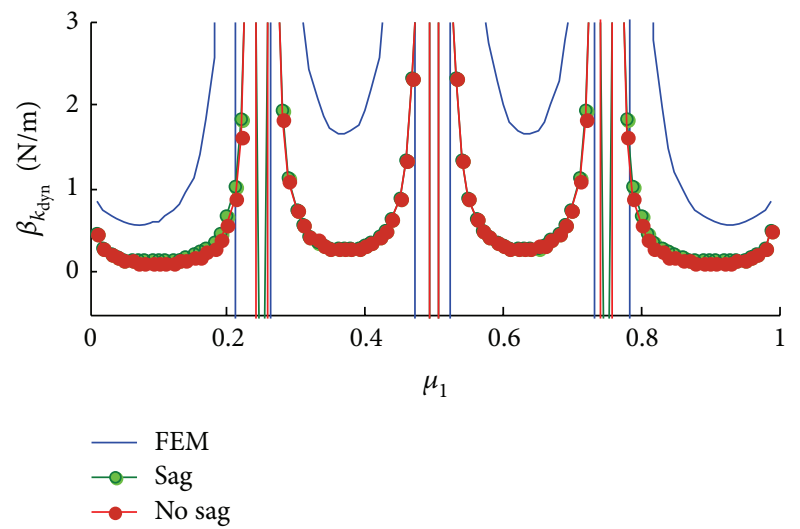

(d) Mode 4

Figure 9: A comparison between the dynamic stiffness of cables with a single transverse degree of freedom using different methods $(\omega=$ $\left.\omega_{1}^{s}, \omega_{2}^{s}, \omega_{3}^{s}, \omega_{4}^{s}\right)$.

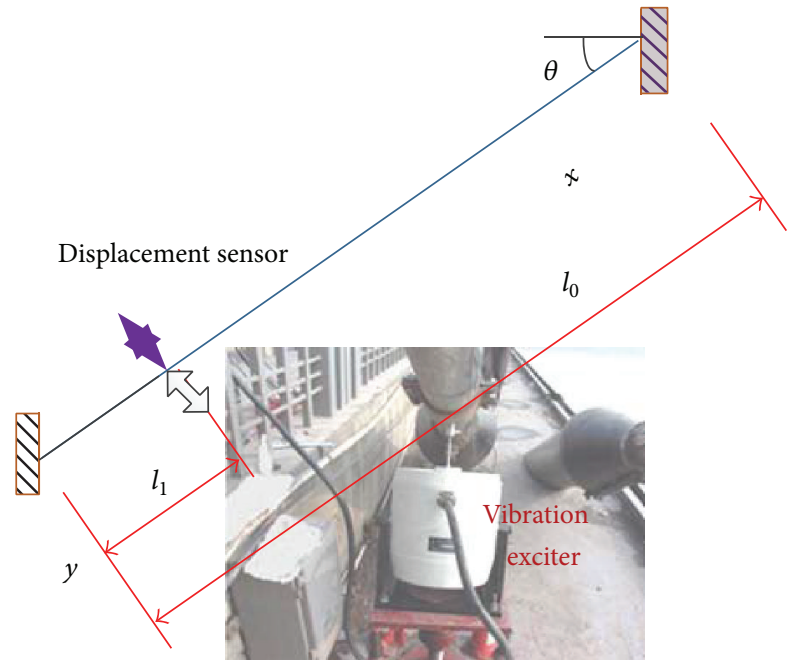

FIGURE 10: Excitation test of a full-scale cable.

become quite different from those obtained from the two methods mentioned previously.

This shows that static condensation method using finite element analysis can be appropriately used as a standard to judge the accuracy of the calculated static stiffness, but not as the standard for dynamic stiffness. In the existing dynamic condensation method using finite element analysis, the stiffness matrix of the model and the mass matrix are reduced to a specified degree of freedom at a certain frequency point (i.e., the modal frequency). The question of whether it is suitable to be considered as a reference for the dynamic stiffness matrix at any frequency still needs to be explored. A new topic is thus proposed, namely, how to realize the reduction of dynamic stiffness at any frequency, a result which would play an important role in the research of the universal dynamic stiffness of structures.

5.3. Vibration Tests of Full-Scale Cables. To further verify the accuracy of the analytical algorithm for the transverse dynamic stiffness matrix provided in this paper, on-site excitation data for a full-scale cable in a cable-stayed bridge are used.

The stay-cable used in the test is $267.3 \mathrm{~m}$ in length and $9.84 \mathrm{~cm}$ in diameter, with an elastic modulus of $2 \mathrm{ell} \mathrm{Pa}$ and a mass per unit length of $76.4 \mathrm{~kg} / \mathrm{m}$. A horizontal tension is applied, and the cable is fixed in the trough, with both ends clamped and secured. A TST-1000 electromagnetic vibration exciter is used at the midpoint of the cable, with power range of $0-1000 \mathrm{~N}$ and a maximum stroke of $\pm 12.5 \mathrm{~mm}$. In 


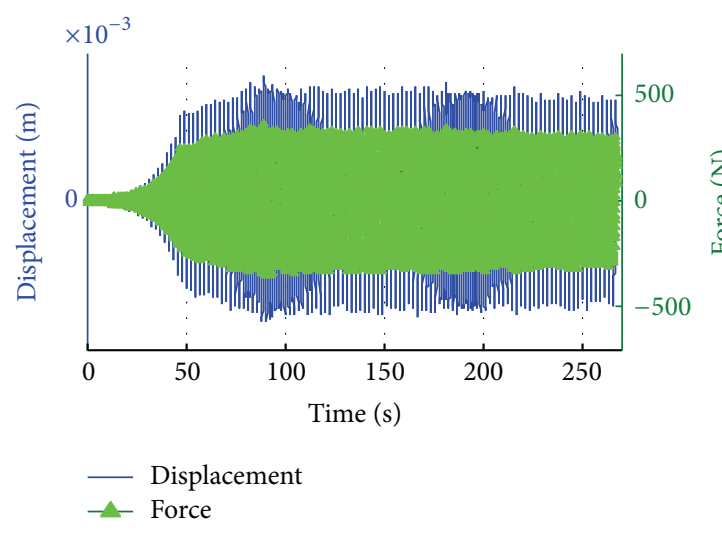

(a)

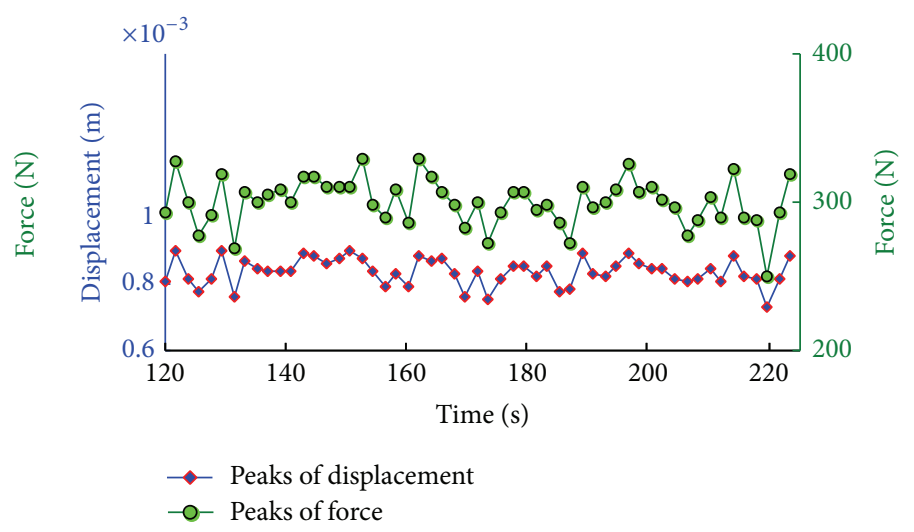

(b)

FIGURE 11: Time history of excitation test and selection of amplitude range.

TABLE 3: A comparison between the calculated and measured dynamic stiffness.

\begin{tabular}{|c|c|c|c|c|c|}
\hline \multicolumn{6}{|c|}{ Frequency } \\
\hline & 1.8632 & 2.0961 & 2.3290 & 2.5619 & 2.7948 \\
\hline Measured & $1.184 \times 10^{5}$ & $7.609 \times 10^{4}$ & $2.275 \times 10^{4}$ & $4.106 \times 10^{4}$ & $1.197 \times 10^{5}$ \\
\hline Method one & $1.157 \times 10^{5}$ & $7.345 \times 10^{4}$ & $2.226 \times 10^{4}$ & $3.983 \times 10^{4}$ & $1.159 \times 10^{5}$ \\
\hline Relative error (\%) & -2.253 & -3.4755 & -2.153 & -3.0075 & -3.195 \\
\hline Method two & $1.077 \times 10^{5}$ & $7.153 \times 10^{4}$ & $2.109 \times 10^{4}$ & $3.958 \times 10^{4}$ & $1.055 \times 10^{5}$ \\
\hline Relative error (\%) & -9.037 & -5.992 & -7.97 & -3.604 & -11.863 \\
\hline Method three & $1.253 \times 10^{5}$ & $8.062 \times 10^{5}$ & $2.153 \times 10^{4}$ & $4.299 \times 10^{4}$ & $1.268 \times 10^{5}$ \\
\hline Relative error (\%) & 5.828 & 5.953 & 5.363 & 4.700 & 5.931 \\
\hline
\end{tabular}

the experiment, a KD9200 resistance displacement meter is used to measure the displacement at the excitation point of the cable. Figure 10 shows the layout and plan of the on-site excitation test.

To begin, the first-order natural frequency is estimated to be $\omega_{1}^{s}=0.3708 * 2 \pi$ using the formula for a taut string. The frequency obtained is multiplied by $0.8,0.9,1,1.1$, and 1.2 , respectively, to be used as five different work conditions in excitation. Figure 11(a) is the time history of the displacement of the excitation point at the midpoint of cable, where the data is acquired by the on-site acquisition device, and the time history of the excitation force is recorded by the excitation device. Figure 11(b) is the curve composed of the maximum amplitude points of stable segments selected from the time histories of displacement and force. The average values are taken to estimate dynamic stiffness.

Equations (42) and (48) are used to calculate the dynamic stiffness matrix of the cable considering (method 1) and neglecting (method 2) sagging effects. Equation (45) is used to calculate the total stiffness of each matrix. Then, (49) is used to obtain the transverse dynamic stiffness with a single degree of freedom, which corresponds to the excitation point of the test. The calculation results are given in Table 3 . The data in the table show, due to the consideration of sagging effects, that method 1 has better precision and yields a result that is closer to the observed value. The maximum error is less than $4 \%$. The method that neglects sagging effects and the finite element method based on static condensation both have larger errors. This result proves that the analytical algorithm of the transverse dynamic stiffness of a cable given in this paper has better precision and reliability.

\section{Conclusion}

The transverse vibration of cables is a primary research focus in the field of cable dynamics. The transverse force element is the most common element in cable structures. The transverse dynamic stiffness of cables is a bridge between transverse vibration and transverse force (excitation). Thus, it is of great significance for the vibration analysis of cable structures and the vibration control of an entire project.

This paper has proposed a dynamic stiffness matrix with two degrees of freedom at any point on a cable (transverse displacement and cross section rotation). On this basis, the dynamic stiffness corresponding to the transverse displacement is derived. Though several factors are simultaneously taken into account, including sagging effects, inclination, flexural stiffness, and boundary conditions, the matrix has a simple form and can be easily realized in programming applications. The existing studies have provided algorithms of dynamic stiffness at the ends of cables, which is incomparable to the results in this study. Compared with the finite element method based on static condensation, our method not only 
achieves a reasonable and accurate result for the static stiffness but also precisely calculates the dynamic stiffness. The variation pattern of the obtained dynamic stiffness is consistent with the findings of the existing studies. Therefore, the proposed method can be used as an effective tool in studying the transverse vibration of cables.

\section{Conflict of Interests}

The authors declare that there is no conflict of interests regarding the publication of this paper.

\section{Acknowledgments}

This paper was sponsored by the Project of National Key Technology R\&D Program in the 12th Five Year Plan of China (Grant no. 2012BAJ11B01), the National Nature Science Foundation of China (Grant no. 50978196), the Key State Laboratories Freedom Research Project (Grant no. SLDRCE09D-01), the Fundamental Research Funds for the Central Universities, and the State Meteorological Administration Special Funds of Meteorological Industry Research (Grant no. 201306102).

\section{References}

[1] E. D. S. Caetano, Cable Vibrations in Cable-Stayed Bridges, vol. 9, IABSE, Zürich, Switzerland, 2007.

[2] N. J. Gimsing and C. T. Georgakis, Cable Supported Bridges: Concept and Design, John Wiley \& Sons, New York, NY, USA, 2011.

[3] E. Rasmussen, "Dampers hold sway," Civil Engineering-ASCE, vol. 67, no. 3, pp. 40-43, 1997.

[4] P. Warnitchai, Y. Fujino, and T. Susumpow, "A non-linear dynamic model for cables and its application to a cablestructure system," Journal of Sound and Vibration, vol. 187, no. 4, pp. 695-712, 1995.

[5] R. W. Clough and J. Penzien, Dynamics of Structures, McGrawHill, New York, NY, USA, 1975.

[6] E. de sa Caetano, Cable Vibration in Cable-Stayed Bridges, IABSE-AIPC-IVBH, Zurich, Switzerland, 2007.

[7] V. Kolousek, Vibrations of Systems with Curved Members, vol. 23, International Association for Bridge and Structural Engineering, Zurich, Switzerland, 1963.

[8] A. G. Davenport, "The wind induced vibration of guyed and self-supporting cylindrical columns," Transactions of the Engineering Institute of Canada, pp. 119-141, 1959.

[9] A. G. Davenport and G. N. Steels, "Dynamic behavior of massive guyed cables," Journal of the Structural Division, vol. 91, no. 2, pp. 43-70, 1965.

[10] A. S. Veletsos and G. R. Darbre, "Free vibration of parabolic cables," Journal of the Structural Engineering, vol. 109, no. 2, pp. 503-519, 1983.

[11] A. S. Veletsos and G. R. Darbre, "Dynamic stiffness of parabolic cables," Earthquake Engineering \& Structural Dynamics, vol. 11, no. 3, pp. 367-401, 1983.

[12] U. Starossek, "Dynamic stiffness matrix of sagging cable," Journal of Engineering Mechanics, vol. 117, no. 12, pp. 2815-2829, 1991.
[13] U. Starossek, "Cable dynamics-a review," Structural Engineering International, vol. 4, no. 3, pp. 171-176, 1994.

[14] A. Sarkar and C. S. Manohar, "Dynamic stiffness matrix of a general cable element," Archive of Applied Mechanics, vol. 66, no. 5, pp. 315-325, 1996.

[15] J. Kim and S. P. Chang, "Dynamic stiffness matrix of an inclined cable," Engineering Structures, vol. 23, no. 12, pp. 1614-1621, 2001.

[16] W. Lacarbonara, "The nonlinear theory of curved beams and fexurally stiff cables," in Nonlinear Structural Mechanics, pp. 433-496, Springer, New York, NY, USA, 2013.

[17] A. Luongo and D. Zulli, "Dynamic instability of inclined cables under combined wind flow and support motion," Nonlinear Dynamics, vol. 67, no. 1, pp. 71-87, 2012.

[18] J. A. Main and N. P. Jones, "Vibration of tensioned beams with intermediate damper. I: formulation, influence of damper location," Journal of Engineering Mechanics, vol. 133, no. 4, pp. 369-378, 2007.

[19] J. A. Main and N. P. Jones, "Vibration of tensioned beams with intermediate damper. II: damper near a support," Journal of Engineering Mechanics, vol. 133, no. 4, pp. 379-388, 2007.

[20] N. Hoang and Y. Fujino, "Analytical study on bending effects in a stay cable with a damper," Journal of Engineering Mechanics, vol. 133, no. 11, pp. 1241-1246, 2007.

[21] Y. Fujino and N. Hoang, "Design formulas for damping of a stay cable with a damper," Journal of Structural Engineering, vol. 134, no. 2, pp. 269-278, 2008.

[22] G. Ricciardi and F. Saitta, "A continuous vibration analysis model for cables with sag and bending stiffness," Engineering Structures, vol. 30, no. 5, pp. 1459-1472, 2008.

[23] H. M. Irvine, "Free vibrations of inclined cables," Journal of the Structural Division, vol. 104, no. 2, pp. 343-347, 1978. 

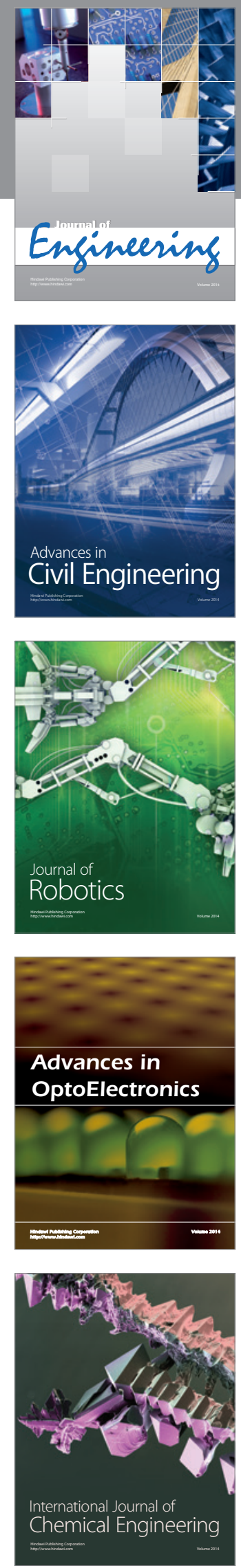

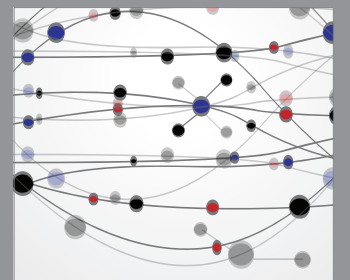

The Scientific World Journal
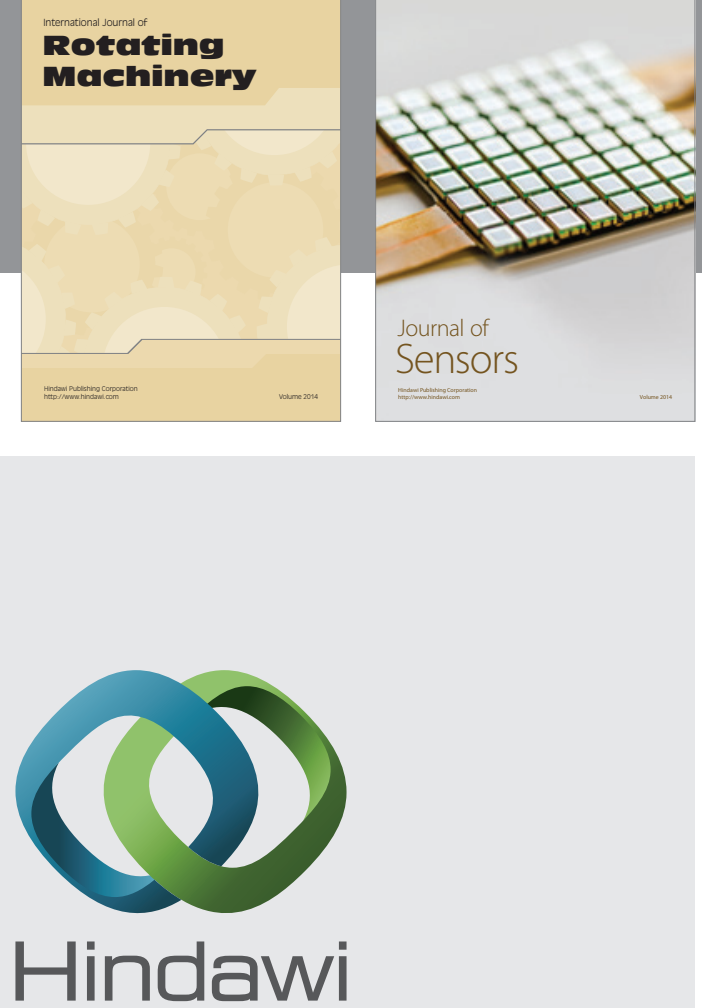

Submit your manuscripts at http://www.hindawi.com
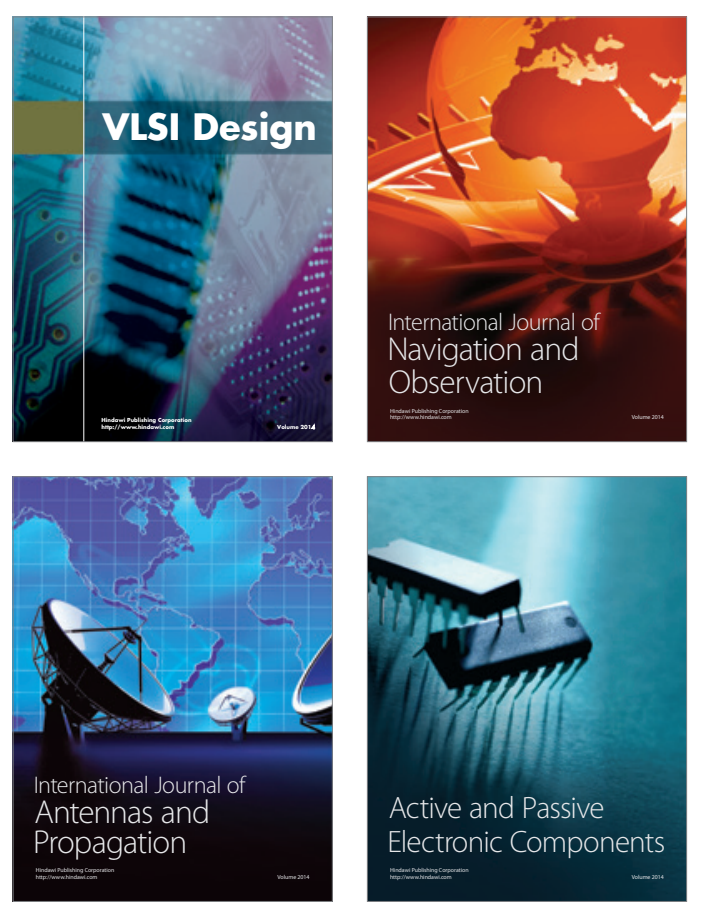
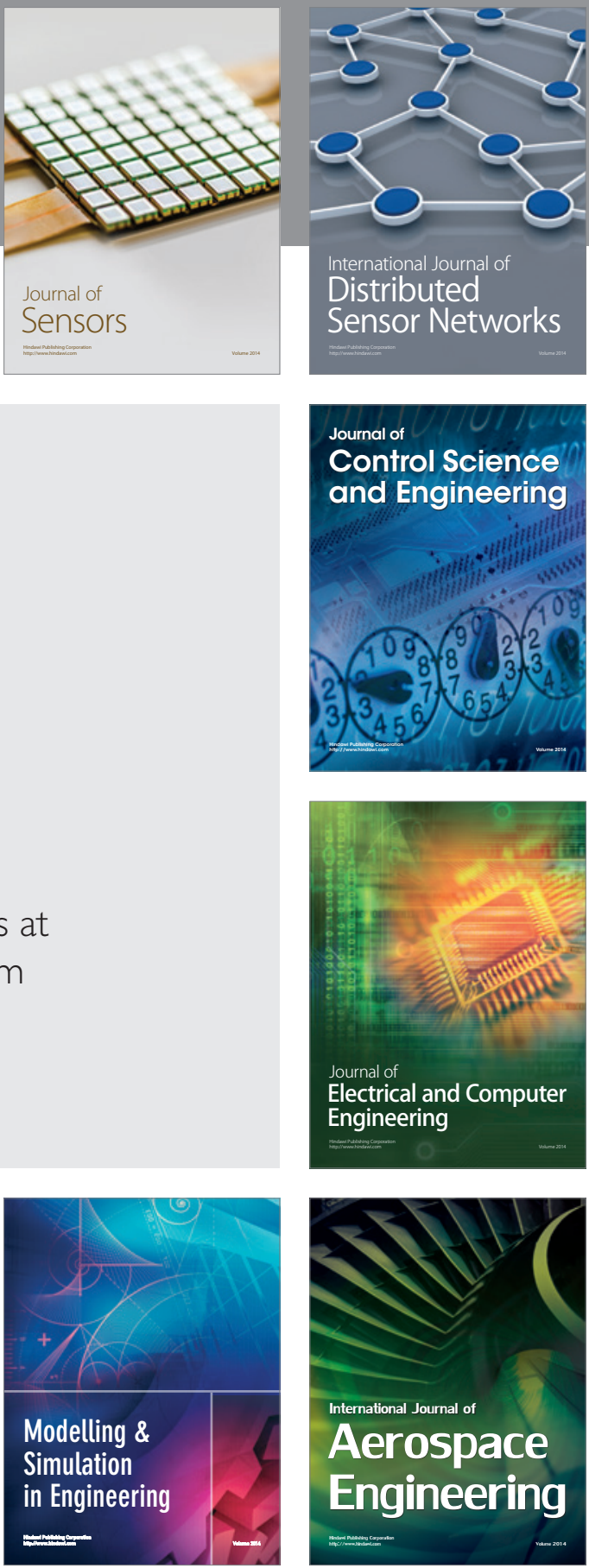

Journal of

Control Science

and Engineering
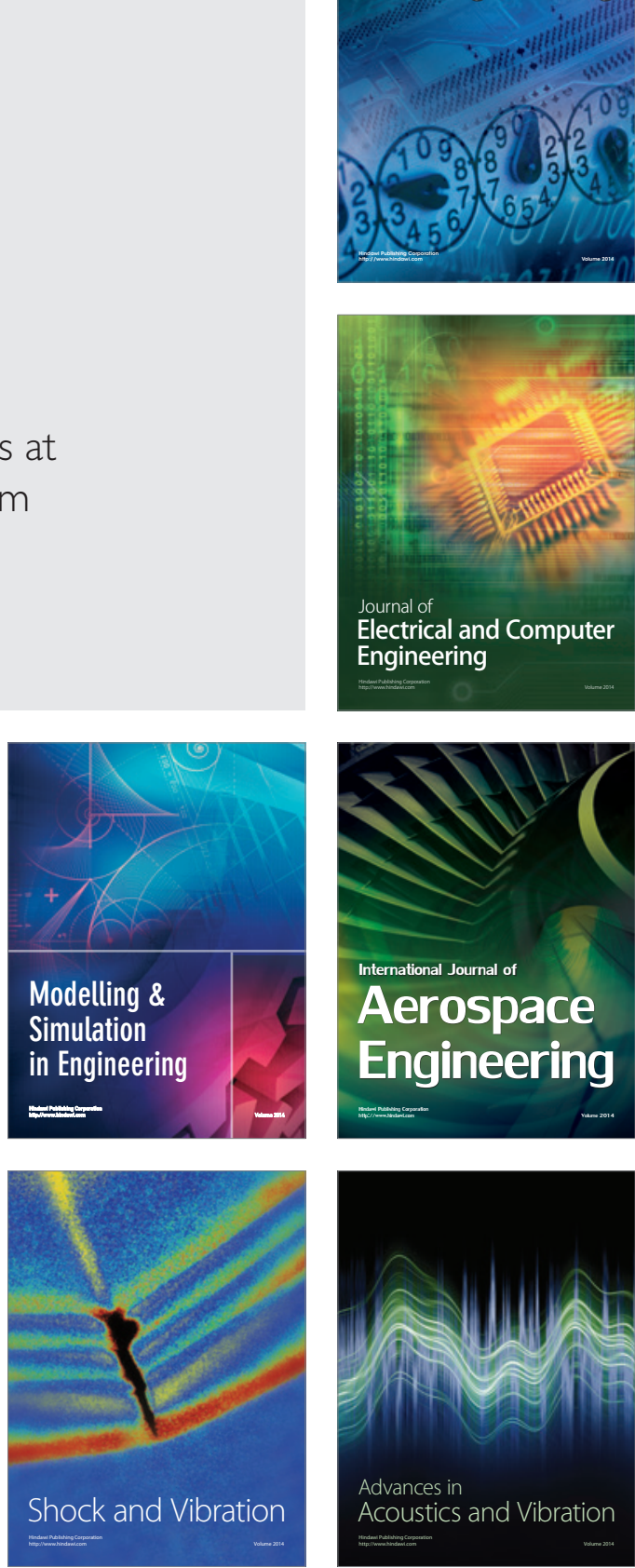